Portland State University

PDXScholar

11-30-1994

\title{
ERP Analysis Using Matched Filtering and Wavelet Transform
}

Xueming Lin

Portland State University

Follow this and additional works at: https://pdxscholar.library.pdx.edu/open_access_etds

Part of the Electrical and Electronics Commons

Let us know how access to this document benefits you.

\section{Recommended Citation}

Lin, Xueming, "ERP Analysis Using Matched Filtering and Wavelet Transform" (1994). Dissertations and Theses. Paper 5065.

https://doi.org/10.15760/etd.6941

This Thesis is brought to you for free and open access. It has been accepted for inclusion in Dissertations and Theses by an authorized administrator of PDXScholar. Please contact us if we can make this document more accessible: pdxscholar@pdx.edu. 


\section{THESIS APPROVAL}

The abstract of the thesis of Xueming Lin for the Master of Science in Electrical Engineering were presented November 30, 1994 and accepted by the thesis committee and the department.

COMMITTEE APPROVALS:

Fu Li, Chair

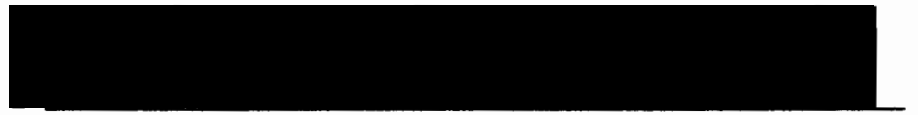

Andrew M. Fraser

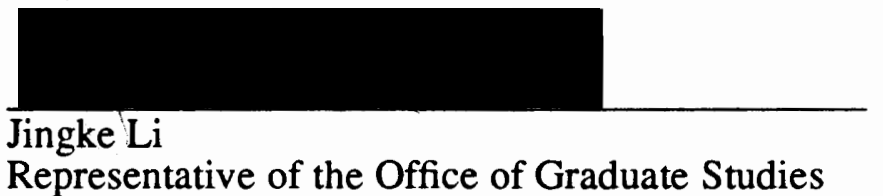

DEPARTMENT APPROVAL:

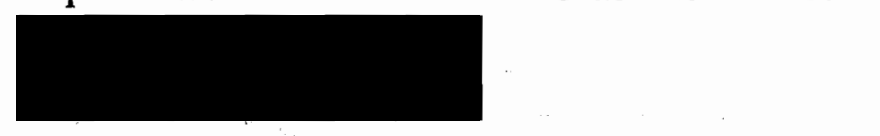

Rolf Schaumann, Chair

Department of Electrical Engineering

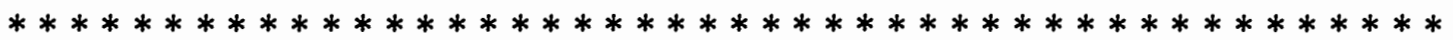

ACCEPTED FOR PORTLAND STATE UNIVERSITY BY THE LIBRARY

by

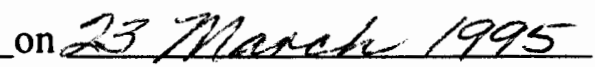




\begin{abstract}
An abstract of the thesis of Xueming Lin for the Master of Science in Electrical Engineering were presented November 30, 1994 and accepted by the thesis committee and the department.
\end{abstract}

Title: ERP Analysis Using Matched Filtering and Wavelet Transform

Event related potentials (ERP's) carry very important information that relates to the performance of the brain functions of the human being. Further studies have identified that one component, in particular, $P_{300}$, is affected by the memory process.

Matched filter is used to improved the SNR of signal ERP's. We use the output of the matched filter to distinguish the difference of the waveforms between normal subjects and memory-impaired subjects. In our study, we found that the peak values of the matched filtering output were different between normal subjects and memoryimpaired subjects.

Also, as an application, wavelet transform is introduced to the ERP analysis. Local maximum of wavelet transform was used as a local feature to find the relationship between the sharp variation points and the memory process.

A comparison between matched filtering and wavelet transform was made and also the correlation coefficients of the peaks and sharp variation points are calculated to find the relationship between the important moments in a memory process. 
ERP ANALYSIS USING MATCHED FILTERING AND WAVELET TRANSFORM

by

XUEMING LIN

A thesis submitted in partial fulfillment of the requirements for the degree of

MASTER OF SCIENCE in

ELECTRICAL ENGINEERING

Portland State University

1995 


\section{ACKNOWLEDGEMENTS}

I wish to express my deepest gratitude to my advisor, $\mathrm{Dr}$. $\mathrm{Fu} \mathrm{Li}$, for his invaluable advice, encouragement and unrelenting patience during the the whole course of my graduate study. Meanwhile, I wish to express my deepest thanks to Dr. Kenneth R. Erickson for his insightful guidance, patience and help during the course of this research.

My sincere appreciation goes to the other members of the committee, Dr. Andrew M. Fraser and Dr. Jingke Li for their helpful comments and understanding. I wish to thank my former teammates, Hong Qi and Dianming Sun, for their valuable preceding research work. Thanks are also due to other members of the faculty and staff at Portland State University who have contributed greatly to my graduate education.

Finally, my parents, my brother Stacey and my girlfriend, deserve my special thanks for their continued support, encouragement and sacrifice throughout. I will forever be indebted to them for all that they have done. 
TABLE OF CONTENTS

PAGE

ACKNOWLEDGMENTS ....................................................................... iii

LIST OF TABLES …................................................................................ vii

LIST OF FIGURES ................................................................................ viii

\section{CHAPTER}

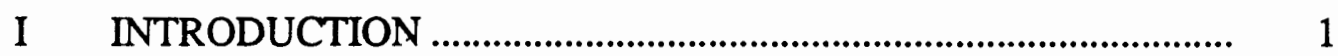

I.1 Thesis Outline .......................................................................... 2

I.2 Notation ....................................................................... 2

II EVENT RELATED POTENTIALS .................................................. 4

II.1 Introduction .............................................................................. 4

II.2 Event Related Potential Waveforms .......................................... 4

II.2.1 ERP Waveforms and EEG Signals ................................... 4

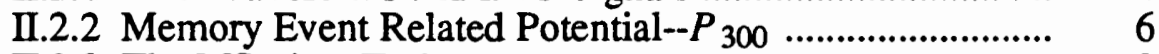

II.2.3 The MSprime Task ........................................................... 9

III ERP ANALYSIS USING MATCHED FILTER _............................... 10

III.1 Introduction ........................................................................... 10

III.2 Matched Filter ........................................................................ 10

III.2.1 The Maximum SNR of The Output ................................. 10

III.2.2 Algorithm ........................................................................ 15

III.3 Matched Filtering of ERP's ...................................................... 17

III.3.1 Design of Matched Filter .............................................. 17

III.3.2 EEG Signal Processing Using The Matched Filter ........ 19

III.3.3 ERP Analysis .................................................................. 22 
III.3.4 Analysis Result .......................................................... 22

IV ERP ANALYSIS USING WAVELET TRANSFORM ................... 25

IV.1 Introduction .................................................................... 25

IV.2 Non-Stationary Signal Analysis ............................................... 25

IV.2.1 Fourier Transform ....................................................... 25

IV.2.2 Short Time Fourier Transform …………………............ 26

IV.3 The Wavelet Transform........................................................ 29

IV.3.1 The Continuous Wavelet Transform ............................ 29

IV.3.2 The Comparison of Wavelet Transform and STFT ....... 31

IV.4 An Implementation of A Particular Wavelet Transform ........ 33

IV.4.1 From the Original Definition to A Simple Form ........... 33

IV.4.2 Dyadic Wavelet Transform ........................................... 34

IV.4.3 Discrete Dyadic Wavelet Transform ………………....... 36

IV.4.4 Fast Implementation of Discrete Algorithms ................ 37

IV.5 Spline Wavelet Transform ...................................................... 41

IV.5.1 Smoothing function ........................................................ 41

IV.5.2 Local Maxima of Spline Wavelet Transform ................. 44

IV.5.3 Local maxima of WT and Regularity of Signal ............. 48

IV.5.4 Daubechies Wavelets

--Another Approch for ERP Analysis ................................. 49

IV.6 EEG Signal Processing Using WT ........................................ 50

IV.6.1 Scale Versus Frequency ............................................... 50

IV.6.2 Detection of ERP's Sharp Variation Point ..................... 52

$\mathrm{V}$ A COMPARISON OF THE WT WITH MATCHED FILTER ........ 58

V.1 Introduction ................................................................. 58

V.2 ERP Analysis Using Correlation Coefficients ....................... 58

V.2.1 Correlation Coefficients ................................................. 58

V.2.2 ERP Analysis ................................................................. 59

V.3 Comparison ......................................................................... 61

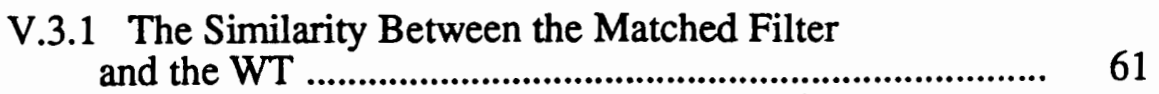

V.3.2 The Differences Between the Matched Filter 63 


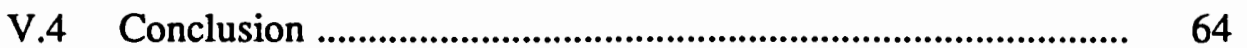

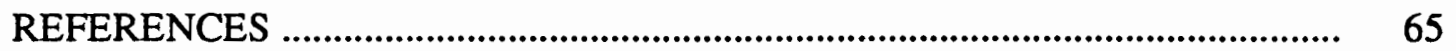




\section{LIST OF TABLES}

TABLE

PAGE

I The ERP Analysis Result after MF ............................................... 24

II The ERP Analysis Result after WT ................................................ 56

III The Means of Time Locations of Sharpest Variation Points and the Mean Time Locations That the Peaks Appear in the Matched Filter ........................................................................... 58

IV Correlation Coefficients between the Outputs of the Matched Filter and Wavelet Transform 61 


\section{LIST OF FIGURES}

FIGURE

PAGE

1. EEG Raw Data.

2. Averaged ERP's of 20 Single EEG Trials of

Low-Tone-High-Tone task.

3. Averaged Normal ERP's of 40 Single EEG Trials

of MSprime task.

4. Averaged Memory-Impaired ERP's of 40 Single

EEG Trials of MSprime Task.

8

5. A Segment of the MSprime Task.

9

6. The Matched Filter for a Physically Realizable

System, Minimum Delay.

7. The Design Procedure of the Matched Filter.

18

8. The Matched Filtering Result.

9. Time-Frequence Plane Corresponding to the Short-

Time-Fourier Transform.

10. Basic Fourier Transform (STFT) and the Wavelet

Transform (WT)

11. Division of the Frequency Domain (a) for the

STFT and (b) for WT.

12. Regions of Influence of a Dirac Pulse at

$t=t_{0}$ for STFT and WT.

13. Block Diagram of the Discrete Wavelet Transform

Implemented with Discrete-Time Filters. 
14. The Family of Spline Functions. ..................................................... 42

15. The Quadratic Spline Wavelet. ........................................................ 43

16. Signal of 256 Sample and Its Dyadic WT. ....................................... 46

17. The Local Maxima of the WT in Figure 15. ...................................... 47

18. The Quadratic Spline Wavelet at Scale $2^{5}$...................................... 51

19. The Sharpest Variation Point of a Latency. ...................................... 52

20. Local Maxima of the Wavelet Transform of EEG Signal at

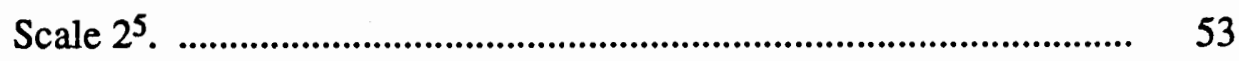

21. The Output of Matched Filter and Smoothed Part of

Wavelet Transform at Scale $2^{5}$. ..................................................... 62 


\section{CHAPTER I}

\section{INTRODUCTION}

Event related potentials (ERP's) carry very important information that relates to the performance of the brain functions of the human being. Further studies have identified that one component, in particular, $P_{300}$, is affected by the memory process. The small amplitudes of ERP's are embedded in the ongoing electroencephalogram (EEG) signal which has an amplitude several times larger than ERP's plus other background noise. Thus, the signal-to-noise ratio (SNR) is less than 1:1 $(0 \mathrm{~dB})$. This small SNR is the one of the most important problems in the field of the analysis of ERP's.

Matched filter is used to improved the SNR of signal ERP's. We use the output of the matched filter to distinguish the difference of the waveforms between normal subjects and memory-impaired subjects.

Wavelet transform has many applications in the signal processing area. Comparing with classic Fourier transform and Short-Time Fourier transform (STFT), it provides a very convenient tool to analyze signals in both the time and frequency domains. In this thesis, a particular wavelet family, the Spline wavelet, is used to detect the sharp variation points of the $P_{300}$ component. The sharpest variation points of the ERP components, clinically, relate to the times at which the maximal number of excitatory or inhibitory processes are being recruited. For instance, the sharper the upslope of the $P_{300}$, the more rapidly the underlying neural networks are being enlisted in producing the signal. The rate of such enlistment (slope) as well as the size of the neural networks enlisted (amplitude) both bear on memory function. We will 
focus on both the slope value and the location where the sharp variation occurs. For other purpose, wavelets can be designed in different ways.

The correlation coefficients of the peaks and the sharp variation points can help in explaining the performance of the memory process.

\section{I.1 THESIS OUTLINE}

This thesis is organized as follows:

Chapter I : General introduction.

Chapter II : A introduction of EEG signals and ERP's . We focus on the component of $P_{300}$ which is related to the memory process.

Chapter III: A review of the theory of the matched filter and the use of ensemble averaging of EEG signals to design a matched filter to improve the signal-to-noise ratio(SNR) of signal ERP's. After matched filtering, we analyze the ERP's by comparing the peak values of the different stimuli. The EEG signals of total 21 trails, 11 for normal subjests and 10 for memory-impaired subjects, are processed. The result shows a fairly clear pattern.

Chapter IV : A comparison of short-time Fourier transform and a multiresolution analysis method--wavelet transform is introduced. The properties of wavelet transform are discussed and particular wavelet family--spline wavelets are used to detect the slope of $P_{300}$ latency.

Chapter V: A comparison between matched filtering and wavelet transform is made in this chapter and also the correlation coefficients of the peaks and sharp variation points are calculated to find the relationship between the important moments in a memory process.

\section{I.2 NOTATION}


$Z$ denotes the set of integers.

$L^{2}$ denotes the Hilbert space of measurable, square-integrable one dimensional functions such that

$$
\int_{-\infty}^{+\infty}|f(x)|^{2} d x<+\infty
$$

We denote the convolution of two functions $f(x) \in L^{2}$ and $g(x) \in L^{2}$ as

$$
f(x) * g(x)=\int_{-\infty}^{+\infty} f(u) g(x-u) d u
$$

The Fourier transform of any signal $f(x)$ is written by $\hat{f}(\omega)$ and is denoted by

$$
\hat{f}(\omega)=\int_{-\infty}^{+\infty} f(x) e^{-i \omega x} d x
$$

For any function $f(x), f_{s}(x)$ denotes the dilation of $f(x)$ by the scale factor $s$

$$
f_{s}(x)=\frac{1}{s} f\left(\frac{x}{s}\right)
$$

For EEG signals and the corresponding outputs of the matched filter and the wavelet transform, 256 sampling points were used to sample each 1.024-second-long sweep, so each sampling point corresponds to 0.004 second.

All EEG signals, including raw data and averaged ERP waveforms, in this thesis are provided by the Erickson Memory Clinic and Research Center. 


\section{CHAPTER II}

\section{EVENT RELATED POTENTIALS}

\section{II.1 INTRODUCTION}

For two decades, brain research has been a very important research issue from which people seek to expose the procedure of the various functions of the human brain and predict the functionally relevant diseases. Being a main research resource, ERP's do carry very important information but the low SNR and the variability of the latencies and amplitudes of the components make obtaining the information very difficult. In this chapter, we introduce in general, ERP waveforms and electroencephalogram (EEG) signals. The memory event related potential-- $P_{300}$ and a visual paradigm designed to elicit $P_{300}$ will be explained further in more detail.

\section{II.2 EVENT RELATED POTENTIAL WAVEFORMS}

\section{$\underline{\text { II.2.1 ERP waveforms and EEG signals }}$}

Event related brain potentials are elicited by the application of sensory stimuli, e.g., visual or auditory, and are of a complicated transient nature characterized by a distinct onset and finite duration.

ERP's are measured using one or more scalp electrodes and most generally referencing the measurements to a body position such as linked ears that is located some distance away from the area of the cortex where the response is expected. The amplitudes of the ERP's vary from tenths of a microvolt to tens of microvolts and are embedded in the ongoing EEG waveform whose amplitude is typically $10-30 \mu \mathrm{V}$. 
Thus, in many instances the signal-to-noise ratio (SNR) is less than 1:1 (0 dB) [1]. It is this small SNR that makes waveform analysis difficult. A segment of raw EEG signal is shown in Figure 1. In this figure, We can not see the ERP waveform due to the noise.
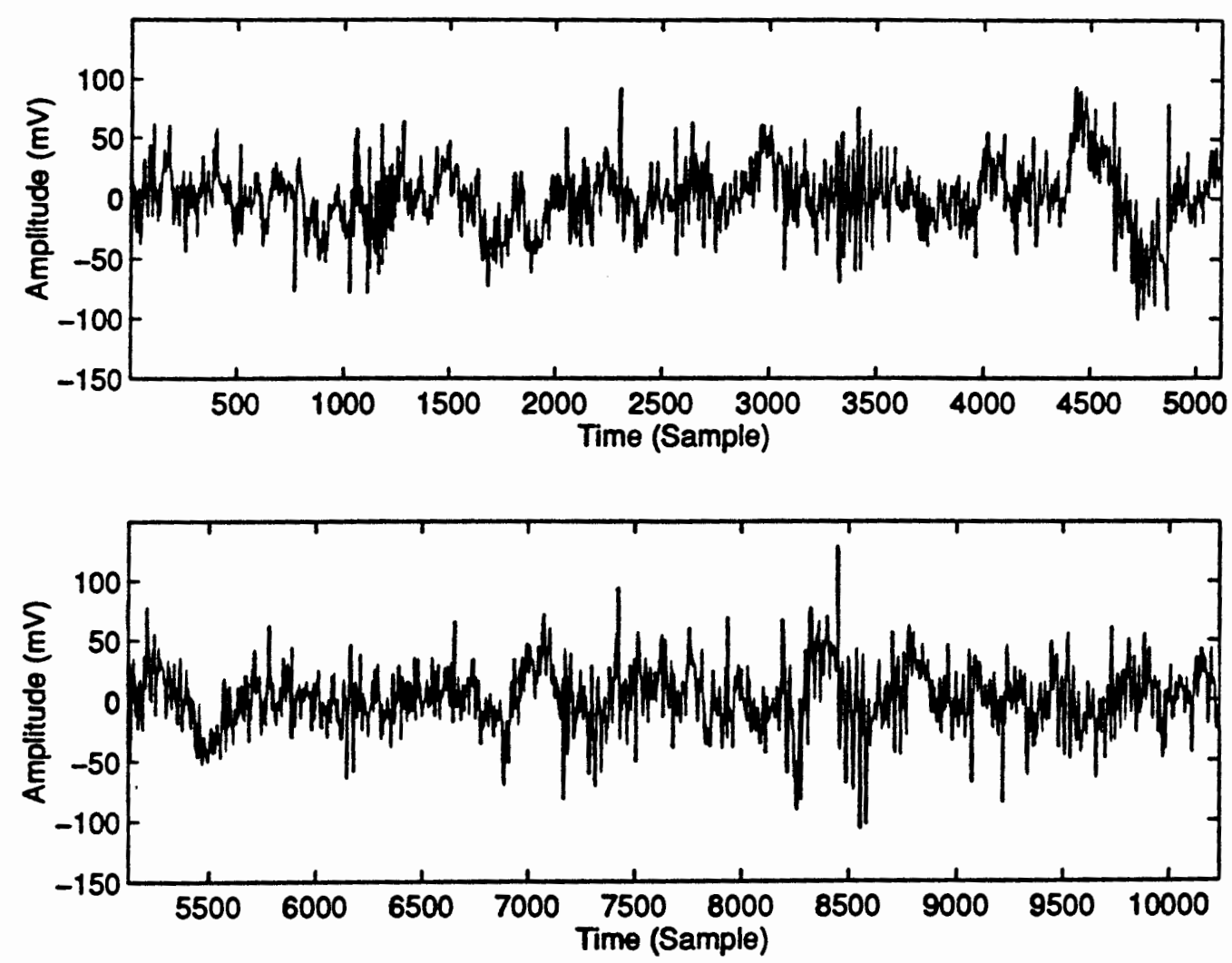

Figure 1. EEG raw data.

Electrophysiologic investigations into cognitive processes during the past two decades have identified certain evoked potential components (termed event-related potentials, or ERPs) which appear sensitive to psychological factors. Figure 2 shows the ERP waveform obtained by averaging 20 single trials of the auditory low-tone- 
high-tone task. Each latency is related to one certain event.

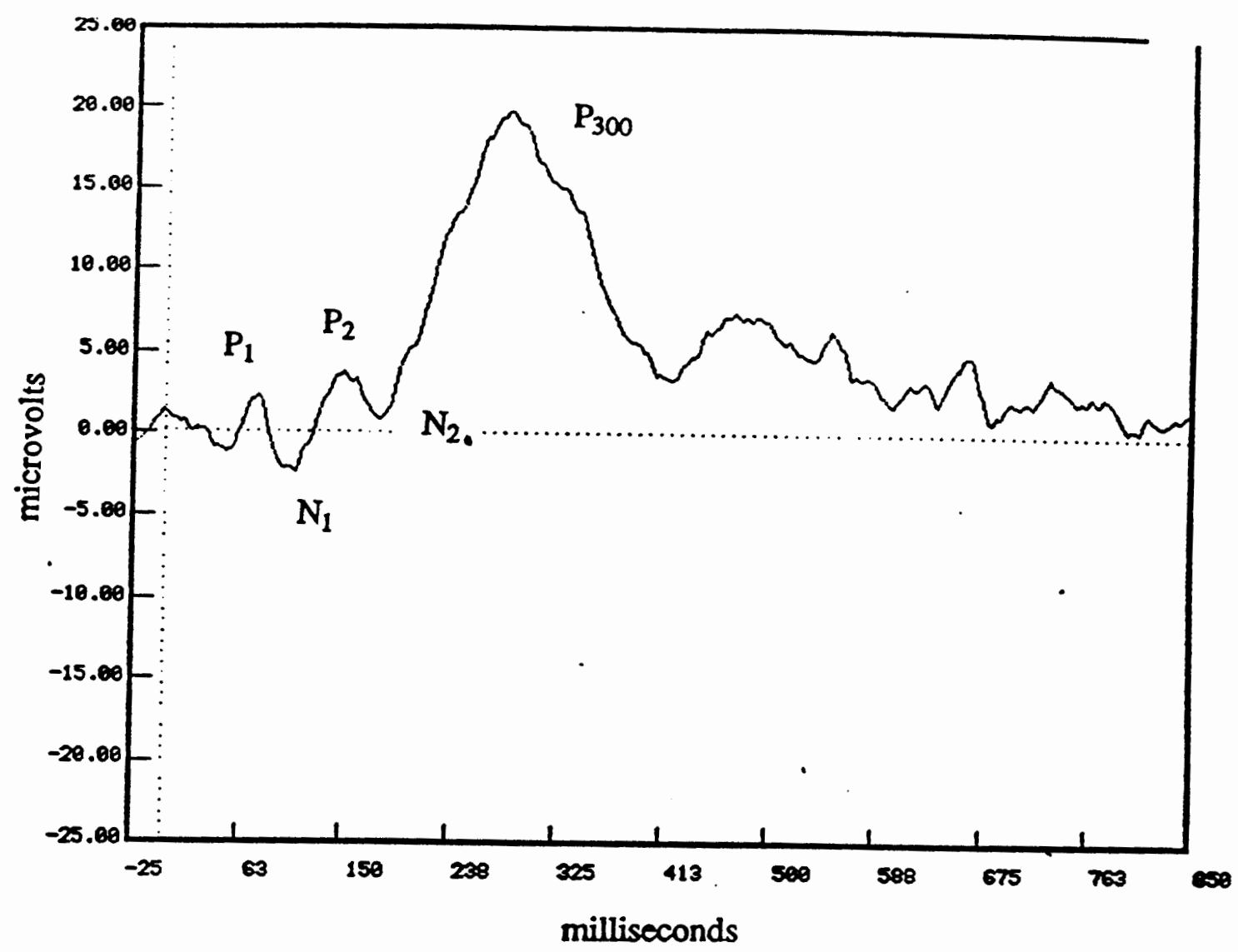

Figure 2. Averaged ERP's of 20 single EEG trials of lowtone-high-tone task.

\section{II.2.2 Memory Event Related Potential-- $P_{300}$}

Of chief interest among these has been the $P_{300}$, a positive waveform peak having its maximum excursion at around 300 milliseconds following stimulus presentation. This has been termed the "expectancy wave" because its amplitudes seem to be inversely related to the frequent stimuli. In other words, it is relatively independent of the particular sensory modality, and largely unaffected by stimulus parameters such as intensity, pitch, color, size, etc. Some investigators have postulated that $P_{300}$ latency is 
a measure of the time required for such processes as stimulus evaluation and categorization. They have found its latency to be relatively independent of processes underlying response selection and execution [2].

A number of studies have observed that the ERP waveform dose not maintain a uniform shape. it differs with respect to scalp distribution. In our study, after careful comparison, we use the data recorded from $\mathrm{Cz}$ [3] (nomenclature is from the International 10-20 System) and the reference point referenced to a common ground, to be our experimented resource. The ERP components have different relationships to stimulus probability and task relevance. Numerous studies have further distinguished

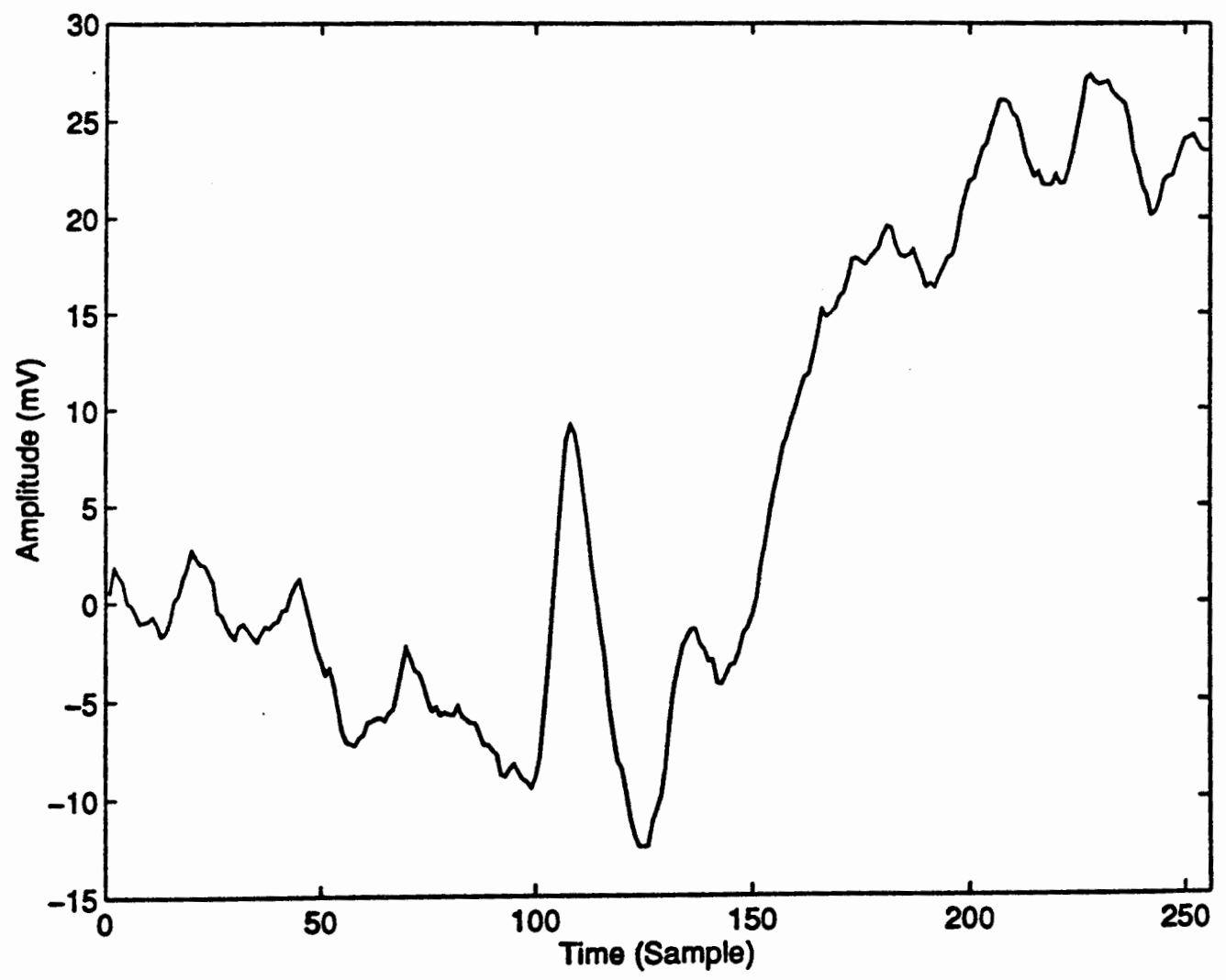

Figure 3. Averaged normal ERP's of $\mathbf{4 0}$ single EEG trials of MSprime task. 
positive components with latencies of $\mathbf{4 0 0}$ milliseconds and beyond with various relationships to processes of stimulus categorization, and response selection and execution. Current nomenclature for the series of components with latencies from 300-600 milliseconds is the "late positive complex". Figure 3 is the averaged waveform of a normal subject for MSprime task and Figure 4 is from a memoryimpaired subject for the same task. We can easily find the difference in the late positive complex between the normal and impaired one. But the $\boldsymbol{P}_{300}$ component is not clear in Figure 3. It is embedded in the late positive complex.

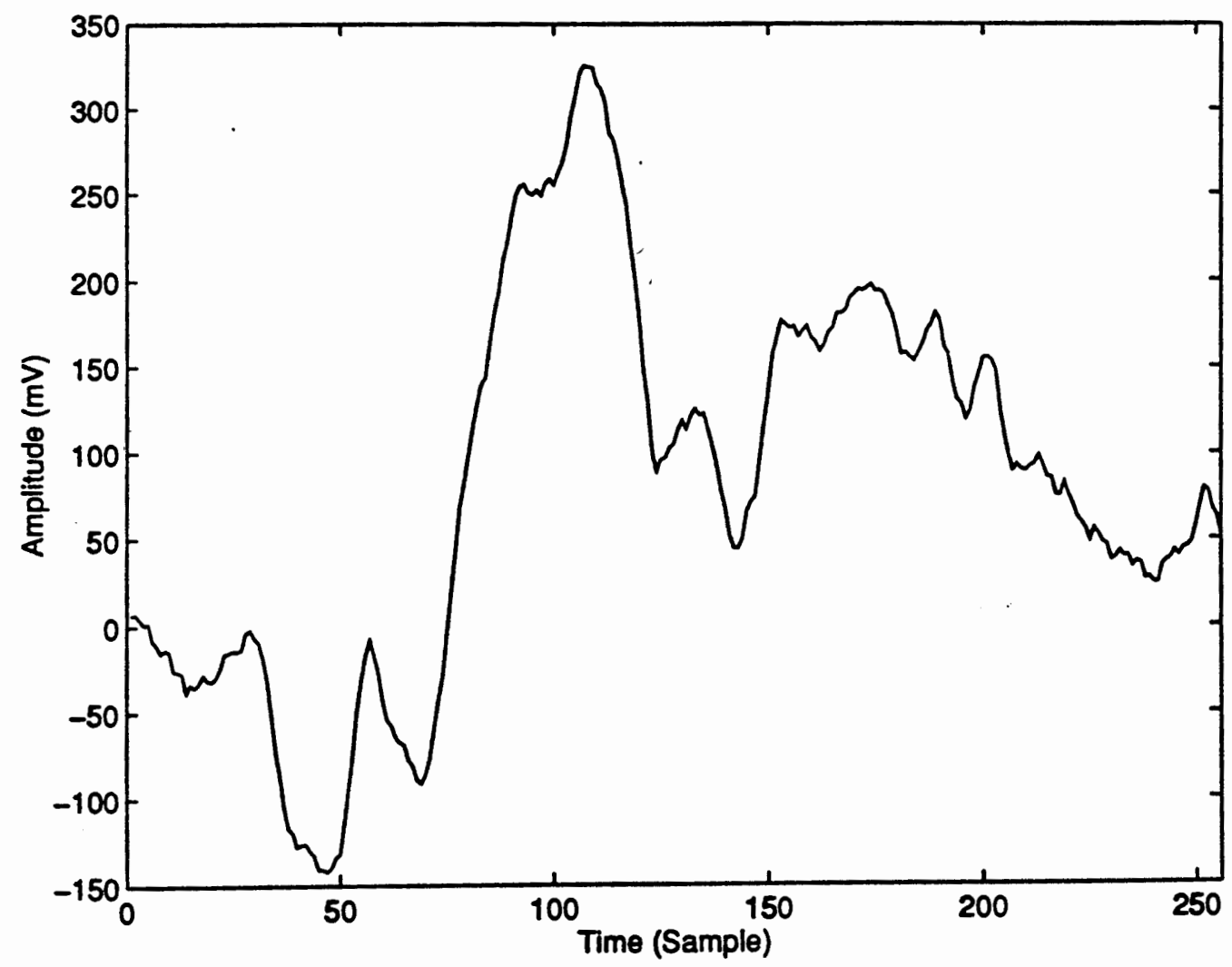

Figure 4 Averaged memory-impaired ERP's of 40 single EEG trials of MSprime task.

The method of averaging EEG signals can show fairly clear patterns for normal 
and impaired ERP waveform. But the real challenge is to identify the waveform that varies significantly between demented and normal subjects from single trials.

\section{II.2.3 MSprime Task}

In our experiment, visual paradigm is designed to elicit $P_{\mathbf{3 0 0}}$ and the other late positive complex components by showing a total of 160 words on the screen. After every three related words, subjects would be asked to judge if the fourth word is related to those three words. So in one complete experiment, there are 40 sets. In each set we call the first three words "background stimuli" and the fourth one "target word". The target words were randomly interspersed to be related or unrelated along the train of 40 sets. Figure 5 shows a example of a segment of the task.

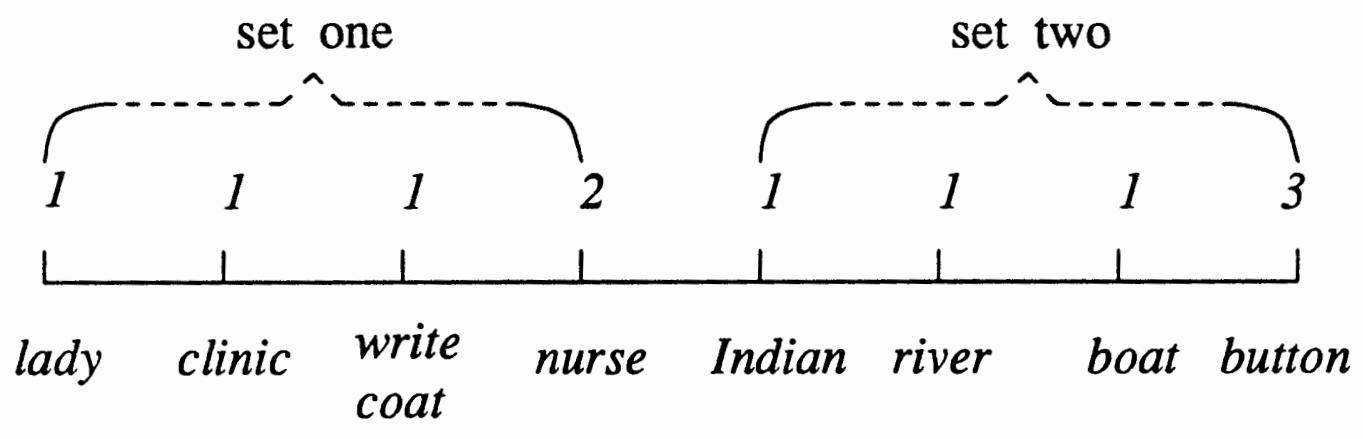

Figure 5. a segment of the MSprime task.

After the first three background words: lady, clinic, and white coat, a beep would indicate to the subject that target word would come next. The fourth one--target word "nurse" is related to the three primming words. For the second set, the target word "button" is not related to its primming words, Indian, river, and boat. For data analysis, we call all the primming words condition 1 , the related words condition 2 , and the unrelated words condition 3. 
In one single ERP trial, a signal is recorded from 200 milliseconds before the stimulus to 824 milliseconds after the stimulus using 256 sampling points(one every 4 millisecond). We call this single segment one sweep. One hundred and sixty 1.024-second-long EEG sweeps are combined into one file.

In every set, we report only the ERP responses elicited by the first of the three primming stimuli among three and the target word. 


\section{CHAPTER IIII}

\section{ERP ANALYSIS USING MATCHED FILTER}

\section{III.1 INTRODUCTION}

Extraction of Event Related Potentials (ERPs) from background EEG is an important issue in brain research [4]. A major problem in the extraction process is the poor Signal-to-noise Ratio (SNR), which characterizes ERP's relative to the ongoing background cerebral activity. The most commonly used method to improve the SNR is ensemble averaging of the signal, time-locked to some external trigger. Many advanced methods that are currently under investigation, apply a variety of adaptive filtering techniques aimed at reducing the number of repetitions, ideally to a single trial. Some are based on an assumed mean behavior of the underlying signal, by which they design optimal filters; others assume a stationary model for ongoing EEG activity, and apply prewhitening techniques to the single trials, hoping to reduce the noise with a minimal signal distortion. However, none of the suggested methods have yet become routine in brain research, due to a high complexity required for some, or only a minor signal improvement for others. In this chapter, we use the matched filtering method to improve the SNR for single trial ERP's and then analyze the ERPs.

\section{2 MATCHED FILTER}

\section{III.2.1 The Maximum SNR of the Output}

Matched filter is a linear time-invariant filter. Matched filter yields a maximum signal-to-noise ratio when the signal with additive noise, if the noise is the white noise, 
passes through it.

Let the signal input to the filter be $[f(t)+n(t)]$, where $f(t)$ is the signal and $n(t)$ is the additive noise. The output of the filter is $\left[f_{o}(t)+n_{o}(t)\right]$ and we wish to maximize the ratio $\left|f_{o}\left(t_{m}\right)\right| / \sqrt{\overline{n_{o}^{2}(t)}}$, where $t=t_{m}$. is the best observation time (to be set). Actually, as we shall see, it tums out to be more convenient to maximize the square of this ratio. So, we are looking for the condition which makes the ratio $\left|f_{o}\left(t_{m}\right)\right|^{2} \overline{n_{o}^{2}(t)}$ have the maximum value.

Let the Fourier transform of $f(t)$ be $F(\omega)$ and let $H(\omega)$ be the frequency transfer function of the desired optimum filter. Then we can write

$$
\begin{aligned}
& f_{o}(t)=\frac{1}{2 \pi} \int_{-\infty}^{+\infty} F(\omega) H(\omega) e^{j \omega t} d \omega, \\
& f_{o}\left(t_{m}\right)=\frac{1}{2 \pi} \int_{-\infty}^{+\infty} F(\omega) H(\omega) e^{j \omega t_{m}} d \omega .
\end{aligned}
$$

The power spectral density of the noise if $S_{n}(\omega)$, so that

$$
\overline{n_{o}^{2}(t)}=\frac{1}{2 \pi} \int_{-\infty}^{+\infty} S_{n}(\omega)|H(\omega)|^{2} d \omega
$$

Dividing the squared magnitude of Equation (1) by (2), we get

$$
\frac{\left|f_{o}\left(t_{m}\right)\right|^{2}}{\overline{n_{o}^{2}(t)}}=\frac{\left.1 \int_{-\infty}^{+\infty} H(\omega) F(\omega) e^{j \omega t_{m}} d \omega\right|^{2}}{2 \pi \int_{-\infty}^{+\infty} S_{n}(\omega)|H(\omega)|^{2} d \omega} .
$$

At this point we make use of the Schwaz inequality:

$$
\left|\int_{-\infty}^{+\infty} f_{1}(x) f_{2}(x) d x\right|^{2} \leq \int_{-\infty}^{+\infty}\left|f_{1}(x)\right|^{2} d x \int_{-\infty}^{+\infty}\left|f_{2}(x)\right|^{2} d x \text {. }
$$


The equality in Equation (4) holds if, and only if,

$$
f_{1}(x)=k f_{2}^{*}(x)
$$

where $k$ is an arbitrary constant.

Now we let the two functions in Equation (4) be identified with $H(\omega) \sqrt{S_{n}(\omega)}$ and $F(\omega) e^{j \omega t_{m}} / \sqrt{S_{n}(\omega)}$, respectively, so that Equation (4) becomes

$$
\left|\int_{-\infty}^{+\infty} H(\omega) F(\omega) d \omega\right|^{2} \leq \int_{-\infty}^{+\infty}|H(\omega)|^{2} d \omega \int_{-\infty}^{+\infty}|F(\omega)|^{2} d \omega
$$

Substitution of this result into Equation (3) gives

$$
\frac{\left|f_{o}\left(t_{m}\right)\right|^{2}}{\overline{n_{o}^{2}(t)}} \leq \frac{\int_{-\infty}^{+\infty}|H(\omega)|^{2} d \omega \int_{-\infty}^{+\infty}|F(\omega)|^{2} d \omega}{2 \pi \int_{-\infty}^{+\infty} S_{n}(\omega)|H(\omega)|^{2} d \omega}
$$

For the special case in which the noise is white, $S_{n}(\omega)=\eta / 2$, and we have

$$
\frac{\left|f_{o}\left(t_{m}\right)\right|^{2}}{\overline{n_{o}^{2}(t)}} \leq \frac{1}{\pi \eta} \int_{-\infty}^{+\infty}|F(\omega)|^{2} d \omega=\frac{E}{\eta / 2},
$$

where $E$ is the energy in $f(t)$ for a $1-0 h m$ load. The equality in Equation (8) holds only if

$$
H_{m}(\omega)=k F^{*}(\omega) e^{-j \omega t_{m}},
$$

or its inverse Fourier transform

$$
h_{m}(t)=k f^{*}\left(t_{m}-t\right)
$$

The constant $k$ is arbitrary and we assume $k=1$ for convenience. And for the real values 


$$
h_{m}(t)=f\left(t_{m}-t\right)
$$

We conclude from this result that the impulse response of the optimum system is the mirror image of the desired input signal $f(t)$, delayed by an interval $t_{m}$. Hence the filter is matched to a particular signal, as conveyed by the terminology matched filter.

$$
g(t)=f(t) * f\left(t_{m}-t\right)
$$

The result expressed in Equation(9) makes good sense intuitively when applied to the magnitude characteristic of a filter so that: $|H(\omega)|=|F(\omega)|$ (Remember that this result was obtained for the case of additive white noise).

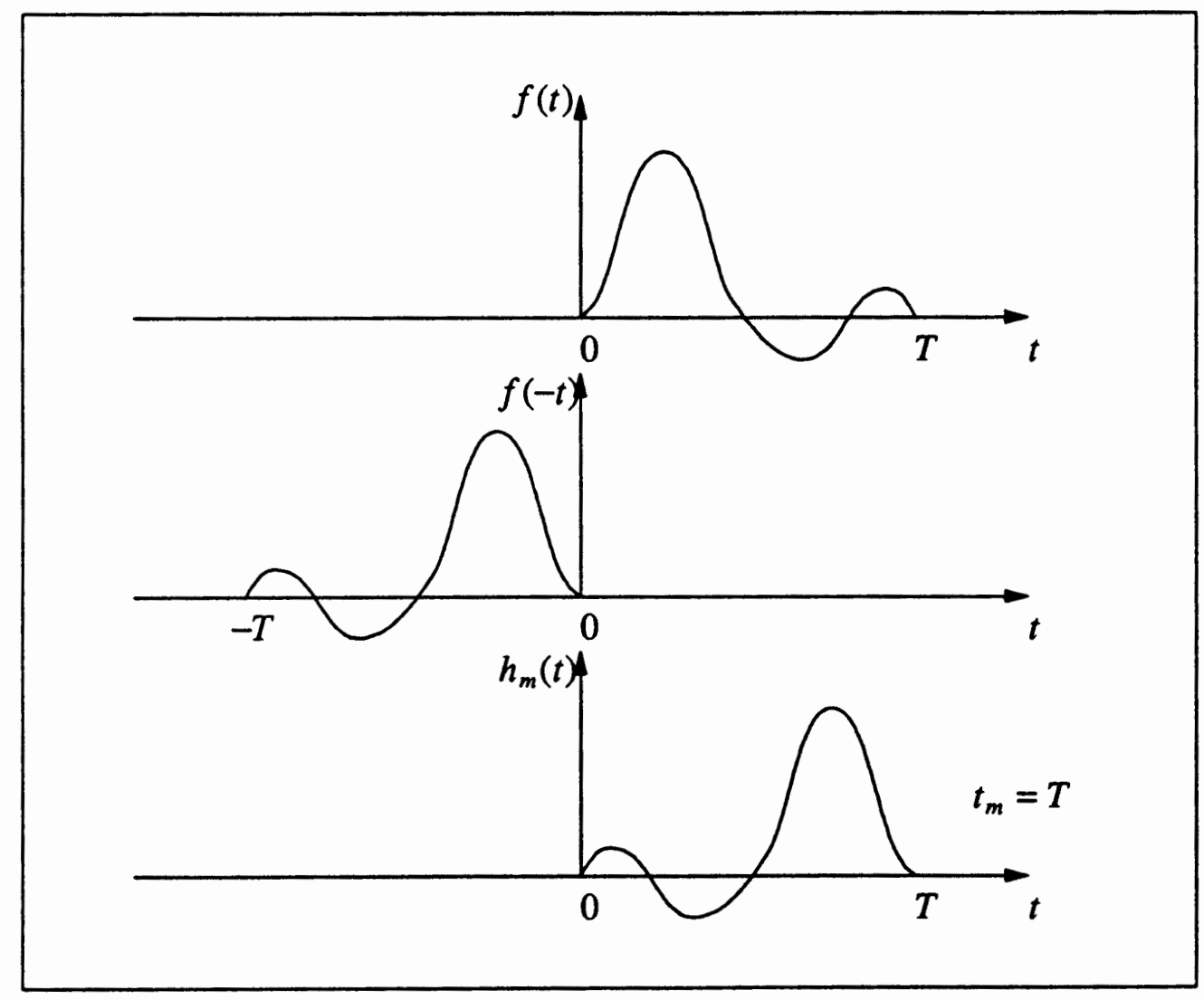

Figure 6. The matched filter for a physically realizable system, minimum delay. 
This result states that one should filter in such a way as to attenuate strongly those frequency components in frequency intervals having little relative signal energy while attenuating very little those components where the relative signal is high [5]. Recall also that we are filtering for signal recognition in the presence of noise, not for signal fidelity (in which one usually desires a "flat" frequency response).

The phase response is also very important and Equation (9) states that the phase shifts in $f(t)$ should be negated in such a way that all frequency components in $f(t)$ add in phase at exactly the time $t=t_{m}$. In contrast, the noise spectral components add with random phases so that the peak-signal-to-rms-noise ratio is maximized.

The signal representation $f(t)$ is assumed to have a finite duration $(0, T)$. The impulse response of the matched filter $f\left(t_{m}-t\right)$ can be obtained by folding $f(t)$ about the vertical axis and shifting it to the right by $t_{m}$ seconds. Restricting consideration to the physically realizable case with minimum delay, we choose $t_{m}=T$. This is illustrated in Figure 6.

At the point $t=t_{m}$, the signal output of the matched filter is given by substituting Equation (9) in Equation (1) with $k=1$ :

$$
f_{o}\left(t_{m}\right)=\frac{1}{2 \pi} \int_{-\infty}^{+\infty}|F(\omega)|^{2} d \omega=E .
$$

Thus the output of the matched filter at $t=t_{m}$ is independent of the particular waveform chosen and depends only on its energy! The mean-square noise output of the matched filter is

$$
\overline{n_{o}^{2}(t)}=E \frac{\eta}{2}
$$

\section{III.2.2 Algorithm}


According to the theory of matched filer, it is a very important step to find the reference signal and then build the matched filter which matches the predicted nature of the signals that we are going to detect. The algorithm extracts the time-varying spectral contents of the reference signal, and uses the information to filter out the noise outside the relevant band for each component of the signals.

The frequency response of the filter is designed to match the frequency content for consecutive time segments of the reference signal. Then, each segment of the signal trial is filtered by the respective reference-based filter. The procedure is applied to a series of signal trials, after which the processed trials are analyzed to obtain the clinically-relevant information.

1. The reference signal is obtained by ensemble averaging of $\mathrm{N}$ signal trials

$$
\bar{s}(n)=\frac{1}{N} \sum_{i=1}^{N} s_{i}(n)
$$

where $s_{i}(n)$ represents each single trial.

2. Find the coefficients of a polynomial $p(x)$ of degree $n$ that fits the reference signal

$$
p(x)=a_{0}+a_{1} x+a_{2} x^{2}+\cdots \cdots+a_{n} x^{n}
$$

The experimental result showed that the 6th degree $p(x)$ carries the most important characteristics of the reference signal.

3. Using those seven coefficients, an approximate reference signal is evaluated as a vector $x(n)$.

4. Fold $x(n)$ about the vertical axis and shift it to the right by the the duration length of this reference signal ( $L=256$ points ). This is the waveform that is going to be used as matched filter $h(n)=x(L-n)$. 
5. As we know in the section III.2., the convolution integral $y(n)$ of $x(n)$ and $h(n)$ has the maximum result at time $t=T$ or $n=L$. It is the same result of the autocorrelation of $x(n)$. So

$$
\begin{aligned}
y_{\max }(n) & =y(L) \\
& =x(n) * h(n) \\
& =\int_{-\infty}^{+\infty} x(\tau) h(L-\tau) d \tau \\
& =\int_{-\infty}^{+\infty} x(\tau) x(\tau) d \tau
\end{aligned}
$$

and $1 / y(L)$ will be used as the normalization coefficient.

\section{III.3 MATCHED FILTERING OF ERP'S}

\section{III.3.1 Design of Matched Filter}

In Clinics, Doctors have run thousand of EEG files, in last two decades, to obtain averaged ERP waveforms. Their experiments report some representative waveforms for normal and memory-impaired subjects. However, those waveforms are not available in electronic format. In our limited data of EEG signals, we found that the averaged ERP waveform of one of the young and normal subjects is the closest one to the previous average for normal subjects in clinics, according to the knowledge of the doctors. The reference signal to design the matched filter in the thesis is obtained by averaging 40 single ERP trials of a young and normal subject, shown as Figure 7(a).

After carefully considering energy distribution of the averaged EEG waveform and comparing its approximate polynomial functions at different 


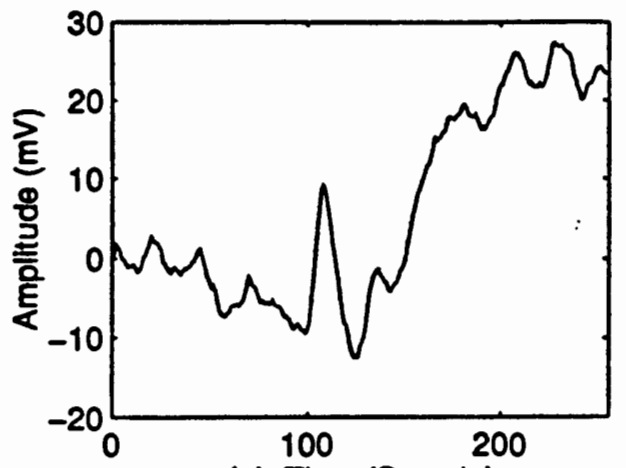

(a) Time (Sample)

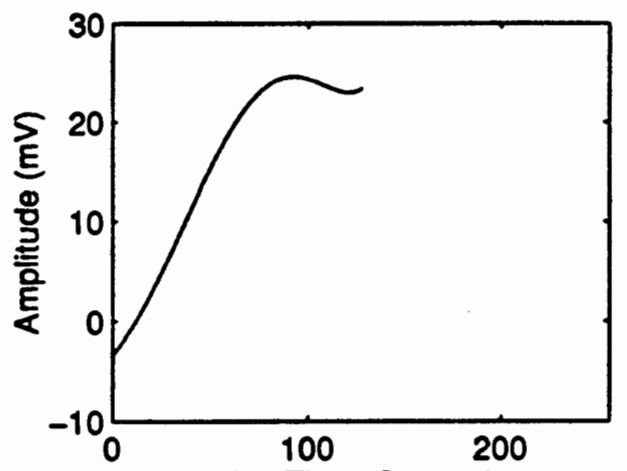

(c) Time (Sample)

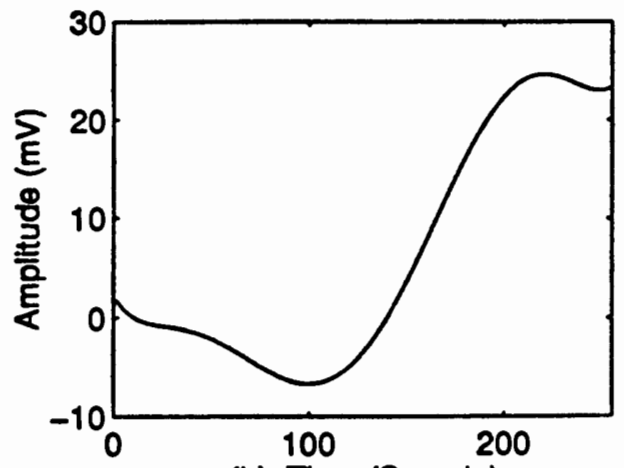

(b) Time (Sample)

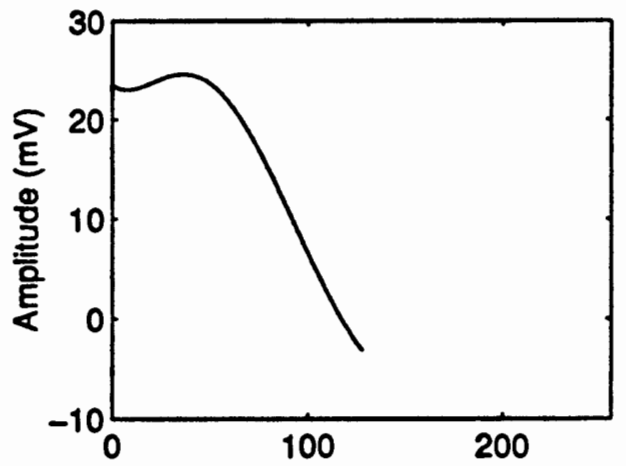

(d) Time (Sample)

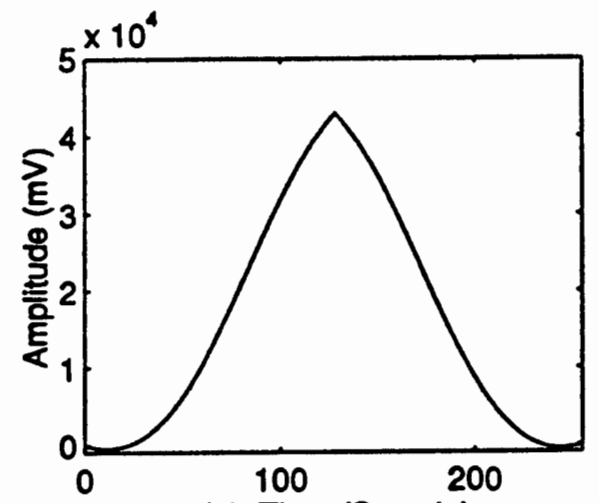

(e) Time (Sample)

Figure 7. The design procedure of the matched filter. 
degrees, we choose a polynomial $p(x)$ of degree 6 to approximately fit the waveform in Figure 7(a). Seven coefficients of the 6th degree polynomial function are calculated

$$
a_{0-7}=[0.0000,-0.0000,0.0000,-0.0031,0.1121,-1.0840,-2.8531] .
$$

Using those seven coefficients, a 6 th degree polynomial function $p(n)$ is evaluated at $1 \leq n \leq 256$, which is shown in Figure 3(b). We found that the values of the first half of this waveform are all very low. That means the first half contains very little energy and information. So we only choose the second half as our characteristic waveform (Shown in Figure 3(c)) which we are going to use to build the matched filter.

Figure $7(\mathrm{~d})$ is the matched filter, after folding and shifting $p(x) 128$ points to the right. Figure $7(\mathrm{e})$ is the auto-correlation of $p(x)$. We choose the maximum value $G$ as the energy normalization constant. where

$$
G=R_{p}(128)=\sum_{n=1}^{128}|p(n)|^{2}
$$

The matched filter output $y(n)$ is obtained by convolving the filters with the signal trial.

\section{III.3.2 EEG Signal Processing Using Matched Filter}

Figure 8.1 (a) is 20 sweeps of EEG raw signal of a normal subject and Figure 8.1 (b) is the output of the matched filter. Figure 8.2 (a) is 20 sweeps of EEG signal of a memory-impaired subject and 8.2 (b) is the matched filter output. We can observe that after matched filtering, the ERP waveforms are very clear. Each strip is for one EEG sweep which contains 256 time points and there is one big peak in almost every sweep. The big peaks whose peak values are beyond the normalization range $[-1,1]$ are considered to be caused by the eyeblinks. 


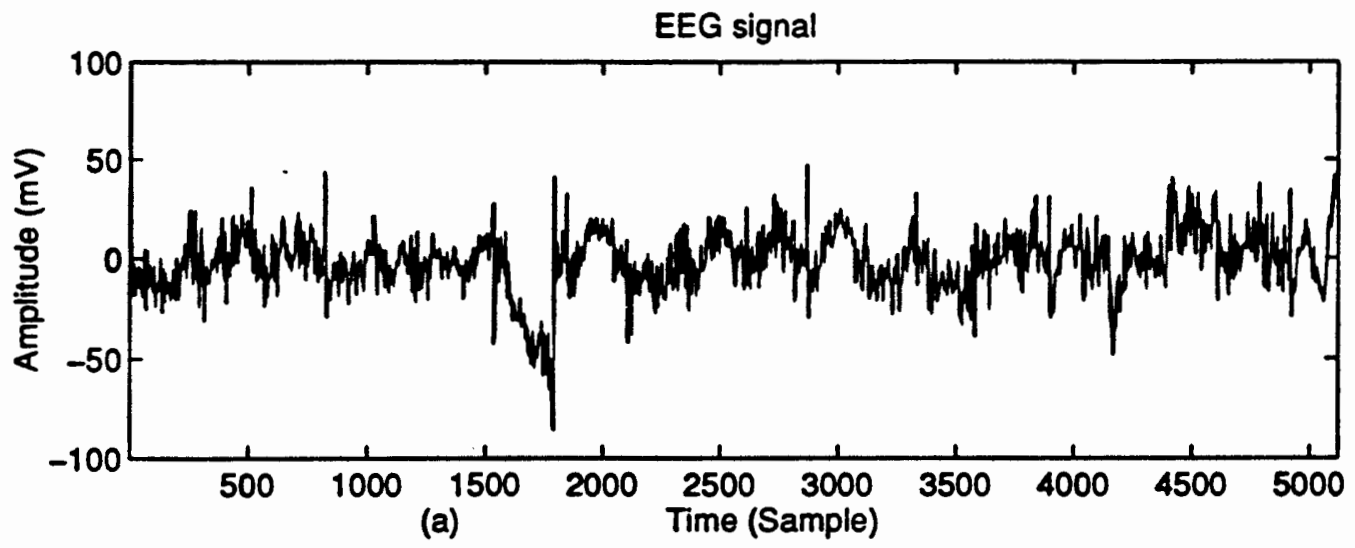

The output of the matched filter

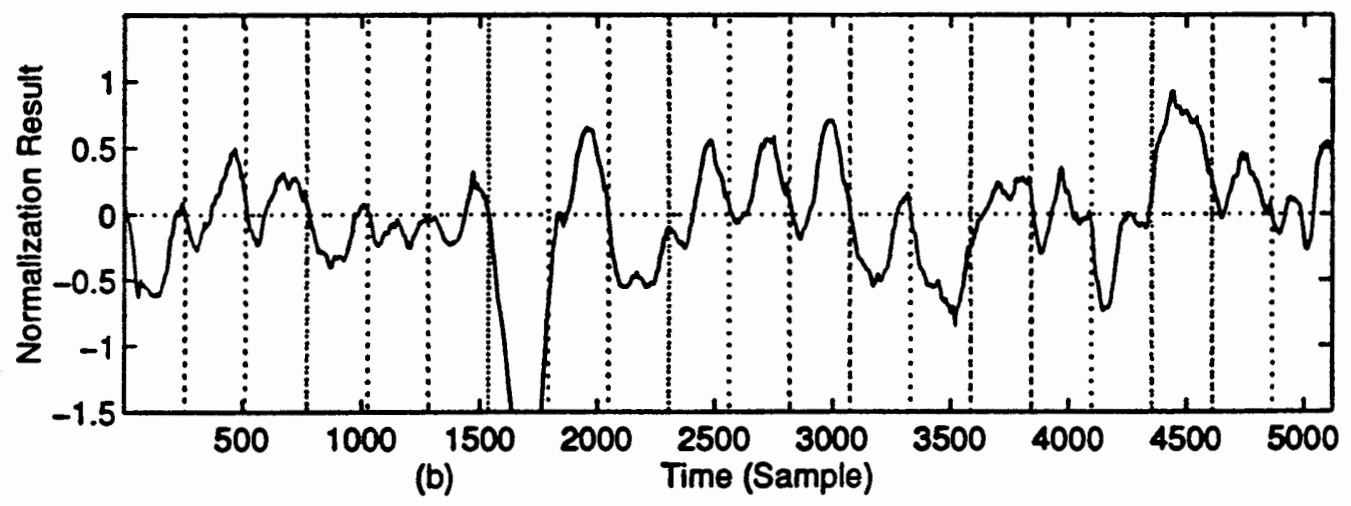

Figure 8.1 The matched filtering result of 20 sweeps of EEG signal of a normal subject. 


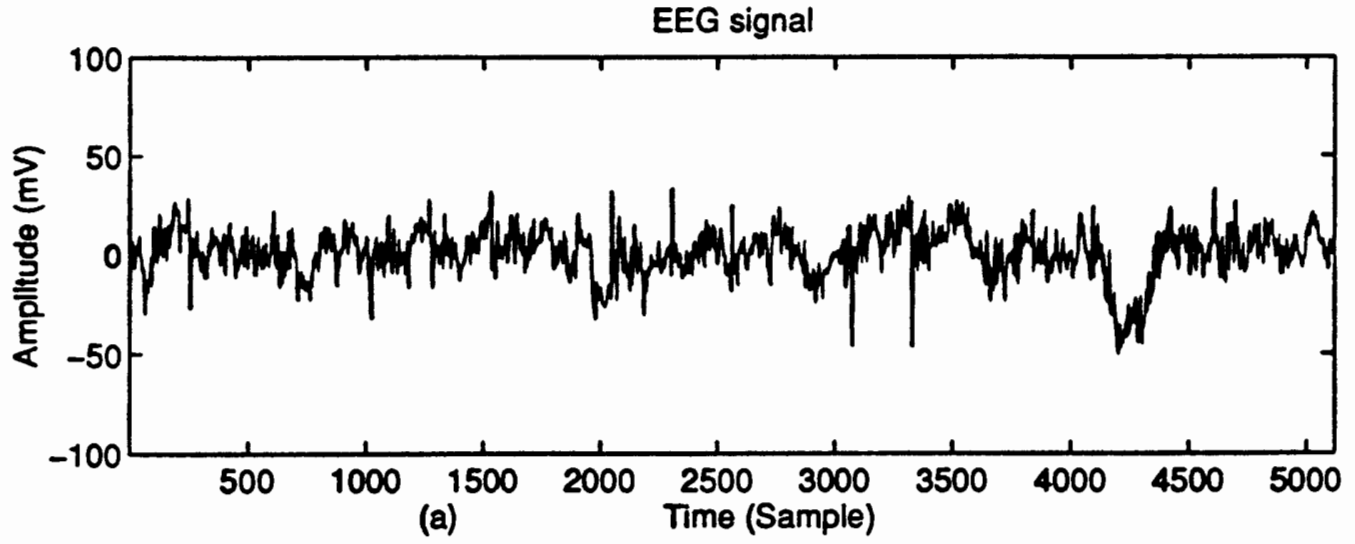

The output of the matched filter

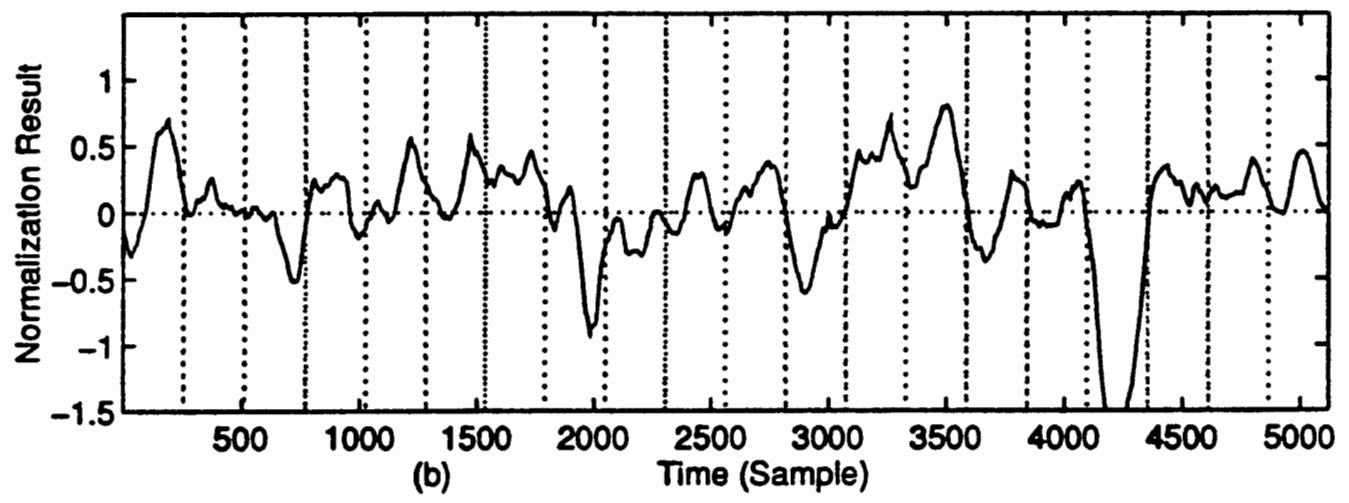

Figure 8.2 The matched filtering result of 20 sweeps of EEG signal of a memory-impaired subject. 


\section{III.3.3 ERP Analysis}

Now we can do the ERP analysis using the output of the matched filter.

1. Find and record the maximum value in each sweep so there are a total of $\mathbf{1 6 0}$ peak values, corresponding to the 160 sweeps. The time latencies where the peaks occur are also saved in a vector for further analysis.

2. In each set, we are only interested in the target word and its first primming word. While being asked to make a judgement on relationship or nonrelationship after three primming works, averaged ERPs suggest that normal subjects have larger ERP responses than impaired subjects.

3. We compare the peak values between each target word (both related and unrelated) and its corresponding first primming word. The value 0.1 , which is the 10 percent of the maximum value of the auto-correlation of the reference signal which was modeled from a normal ERP waveform, is chosen as the threshold. Medically, there is significant difference between two conditions. For conditions 2 and conditions 3 respectively, the percentage that the peak values are higher than that of their first condition 1 is computed.

4. If either condition 1 or target condition is eyeblink, the comparison will not be made in that set. The normalization helps us to remove the peak values that were cause by the eyeblinks but it cause some error. We will discuss it later.

\section{III.3.4 Analysis Result}

From the Table 1, we can observe that for normal subjects, there are only two exceptions, the trial I of subject 01 and trial I of subject 05 , whose percentages are below $40 \%$. All others are above $40 \%$ for both condition 2 and condition 3 , and for impaired subjects, the percentages for both condition 2 and condition 3 are obviously lower than the normal subjects. Only trial II of subject 06 and trial II of subject 08 are 
the exceptions. Their percentages are higher than $40 \%$. Totally, 18 trials are processed and the experimental accuracy is $(18-4) / 18=77.8 \%$

We noticed that for few cases, using normalization to detect the eyeblinks is not very accurate. In one hand, for some memory-impaired subjects, the waveforms are very low, even some eyeblink peaks are still in the range of normalization. and on the other hand, for some very active normal subjects, some regular responses are out of the range. Even though those are just a few cases, they did cause some error to our result. 


\begin{tabular}{|c|c|c|c|c|}
\hline Group & Subject & Trial & Conditoin $2(\%)$ & Condition $3(\%)$ \\
\hline \multirow{9}{*}{ Normal } & 01 & I & 32 & 33 \\
\hline & & II & 55 & 78 \\
\hline & 02 & I & 45 & 56 \\
\hline & & II & 45 & 83 \\
\hline & 03 & I & 55 & 40 \\
\hline & & II & 45 & 33 \\
\hline & 04 & I & 41 & 50 \\
\hline & & II & 53 & 79 \\
\hline & 05 & I & 23 & 39 \\
\hline \multirow{10}{*}{ Impaired } & 06 & I & 31 & 27 \\
\hline & & II & 68 & 55 \\
\hline & 07 & I & 20 & 15 \\
\hline & & II & 19 & 33 \\
\hline & 08 & I & - & - \\
\hline & & II & 45 & 56 \\
\hline & 09 & I & 20 & 5 \\
\hline & & II & 14 & 6 \\
\hline & 10 & I & 23 & 28 \\
\hline & 11 & I & 18 & 30 \\
\hline
\end{tabular}

Table 1. The ERP analysis result using matched filter. 


\section{CHAPTER IV}

\section{WAVELET AND WAVELET TRANSFORM}

\section{IV.1 INTRODUCTION}

The wavelet transform (WT) is a tool that cuts up data for functions or operators into different frequency components, and then studies each component with a resolution matched to its scale. It gives a better point of view to signal analysis than the classical short-time Fourier transform (STFT). The wavelet transform of signal which evolves in time depends on two variables: scale (or frequency) and time; wavelets provide a method for time-frequency localization.

For some particular wavelet functions, the local maxima of the wavelet transform correspond to the sharp variation points of the signal. Points of sharp variations are found often carrying the important information for analyzing the properties of signal and image.

In this chapter, for a clear way to understand wavelet transform, we need go back to the Fourier transform and short-time Fourier transform first. From the comparison of short-time Fourier transform and wavelet transform, we will explain why we chose wavelet transform for EEG signal analysis. Then the spline wavelets are introduced. We explain how the spline wavelets can detect the sharp variation points and how they work for signal ERP's.

IV.2 NON-STATIONARY SIGNAL ANALYSIS

\section{IV.2.1 Fourier Transform}


For stationary signals, that is, a signal whose properties do not evolve in time, $f(t)$, we have "stationary transform"--Fourier transform:

$$
F(\omega)=\int_{-\infty}^{+\infty} f(t) e^{-i \omega t} d t
$$

The analysis coefficients $F(\omega)$ define the notion of global frequency $\omega$ in a signal. Analysis works well if the signal is composed of a few stationary components. However, any abrupt change in time in a non-stationary signal is spread out over the whole frequency axis in $F(\omega)$. Therefore, an analysis adapted to a nonstationary signal requires more than the Fourier transform.

The idea is to introduce a "local frequency" parameter (local in time) so that "local" Fourier transform looks at the signal through a window over which the signal is approximately stationary.

\section{IV.2.2 Short-Time Fourier Transform}

The "instantaneous frequency" has often been considered as a way to introduce frequency dependence on time. If the signal is not narrow-band, however, the instantaneous frequency averages different spectral components in time. To become accurate in time, we therefore need a two-dimensional time-frequency representation $S(t, \omega)$ of the signal $f(t)$ composed of spectral characteristics depending on time, the local frequency $\omega$ being defined through an appropriate definition of $S(t, \omega)$.

Consider a signal $f(t)$, and assume it is stationary when seen through a window $g(t)$ of limited extent, centered at time location $\tau$. The Fourier transform of window signals $f(x) g^{*}(x-\tau)$ yields the short-time Fourier transform [6]:

$$
\operatorname{STFT}(\tau, \omega)=\int_{-\infty}^{+\infty} f(t) g^{*}(t-\tau) e^{-i \omega t} d t
$$


which maps the signal into a two-dimensional function in a time-frequency $(\tau, \omega)$.

The parameter $\omega$ in Equation (17) is similar to the Fourier frequency and depends critically on the choice of the window $g(t)$.

Figure 9 shows vertical stripes in the time-frequency plane, illustrating this "windowing of the signal" view of the STFT. This plane can be seen either as a succession of Fourier transforms of a windowed segment of the signal (vertical stripes) or as a modulated analysis filter band (horizontal stripes). An alternative view is based on a filter bank interpretation of same process, At a given frequency $\omega$, Equation (22) amounts to filtering the signal "at all time" with a bandpass filter having as impulse response the window function modulated to that frequency.

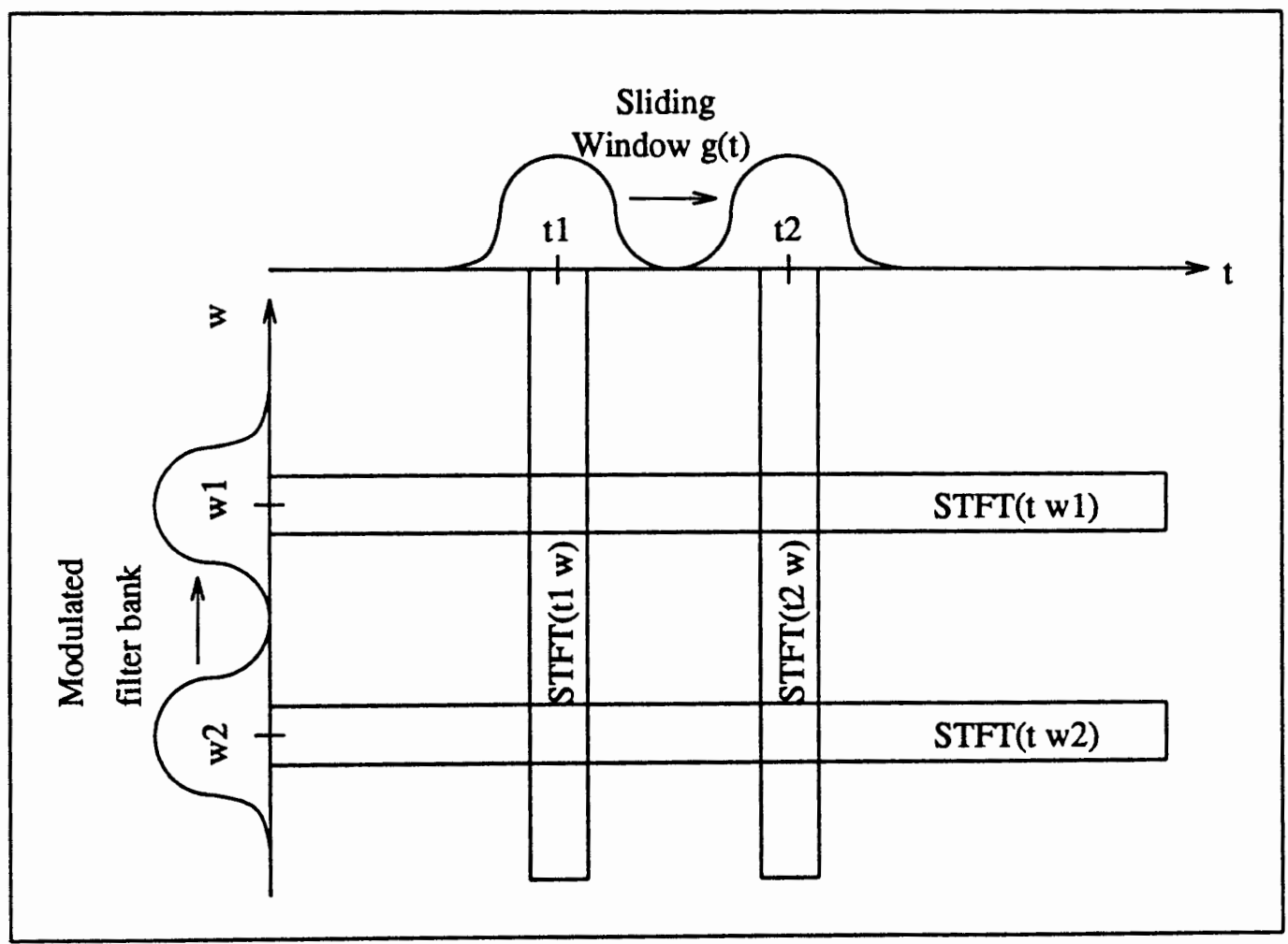

Figure 9.Time-frequence plane corresponding to the shorttime Fourier transform. 
From this dual interpretation, a possible drawback related to the time and frequency resolution can be shown: Consider the ability of the STFT to discriminate between two pure sinusoids. Given a window function $g(t)$ and Fourier transform $G(\omega)$ define the bandwidth $\Delta \omega$ of the filter as:

$$
\Delta \omega^{2}=\frac{\int_{-\infty}^{+\infty} \omega^{2}|\hat{g}(\omega)|^{2} d \omega}{\int_{-\infty}^{+\infty}|\hat{g}(\omega)|^{2} d \omega}
$$

where the denominator is the energy of $g(t)$. Two sinusoids will be discriminated only if they are more than $\Delta \omega$ apart. Thus, the resolution in frequency of the STFT analysis is given by $\Delta \omega$. Similarly, the resolution in the time is given by $\Delta t$ as

$$
\Delta t^{2}=\frac{\int_{-\infty}^{+\infty} t^{2}|\hat{g}(t)|^{2} d t}{\int_{-\infty}^{+\infty}|\hat{g}(t)|^{2} d t}
$$

where the denominator is again the energy of $g(t)$. Two pulses in time can be discriminated only if they are more than than $\Delta t$ apart.

Now, resolution in the time and frequency cannot be arbitrarily small, because their product is lower bounded.

$$
\Delta t \Delta \omega \geq \frac{1}{2}
$$

It means that one can only trade time resolution for frequency, or vice versa. More important is that once a window has been chosen for the STFT, then the timefrequency resolution given by Equations (22) and (23) is fixed over the entire timefrequency plane (since the same window is used at all frequencies). For example, if the signal is composed of small bursts associated with long quasi-stationary 
components, then each type of component can be analyzed with good time resolution or frequency resolution, but not both.

\section{IV.3 THE WAVELET TRANSFORM}

\section{IV.3.1 The Continuous Wavelet Transform}

To overcome the resolution limitation of the STFT, one can imagine letting the resolution $\Delta t$ and $\Delta \omega$ vary in the time-frequency plane in order to obtain a multiresolution analysis. Intuitively, when the analysis is viewed as a filter bank, the time resolution must increase with the central frequency of the analysis filter. We therefore impose that $\Delta \omega$ is proportional to $\omega$ or

$$
\frac{\Delta \omega}{\omega}=c
$$

where $c$ is a constant.

When Equation (26) is satisfied, we see that $\Delta \omega$ and therefore also $\Delta t$ changes with the center frequency. It seems that we use the shorter windows at higher frequencies and the longer windows at lower frequencies. Of course, they still satisfy the Heisenberg inequality Equation (19).

From Figure 10, we can see that in the case of STFT, no matter how high or how low the frequence is, the time window is the same and for WT, window does change with frequency; the lower frequency, the wider window or vice versa. But now, the time resolution becomes arbitrarily good at higher frequencies, while the frequency resolution becomes arbitrarily good at lower frequencies. For example, two very close short bursts can always be eventually separated in the analysis by going up to higher analysis frequencies in order to increase time resolution (see Figure 11(b)). This kind of analysis of course works best if the signal is composed of high frequency components of short duration plus low frequency components of long duration, which 
is often the case with signals encountered in practice.

a)

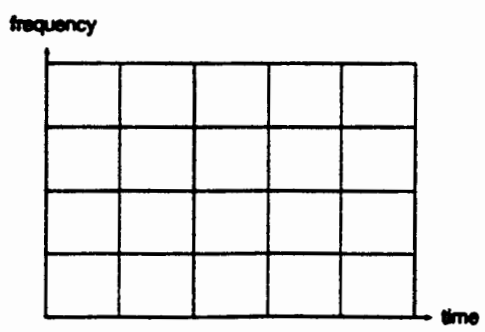

c)

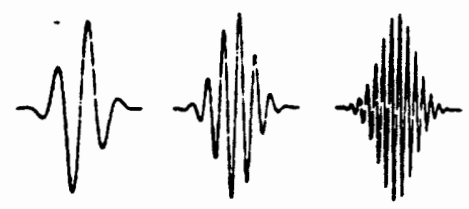

b)

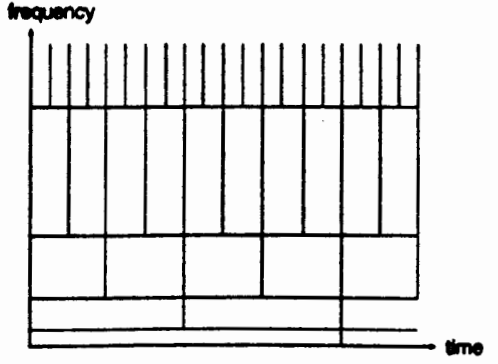

d)

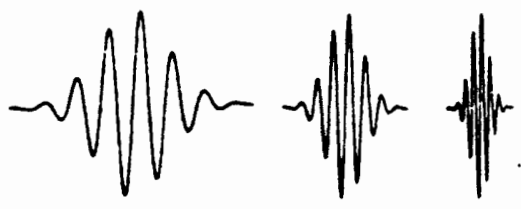

Figure 10. Basic function and time-frequency resolution of the STFT and the WT. (a) Coverage of the time-frequency plane for the STFT, (b) for the WT, (c) corresponding basic function form the STFT, and (d) the basic function for the WT (wavelets).

A generation of the concept of changing resolution at different frequencies is obtained with so-called wavelet packets [6], where arbitrary time-frequency resolutions are chosen depending on the signal. The continuous wavelet transform exactly follows the above ideas.

For a signal $h(t)$, then $h(s t)$, where $s>0$, is contracted if $s>1$ and expanded if $s<1$. The wavelet transform for signal $f(t)$ can be written as [7]

$$
W_{s} f(t)=\frac{1}{\sqrt{s}} \int f(t) h\left(\frac{t-x}{s}\right) d t
$$

It presents that as the scale $s$ increases, the window function $h(t-x / s)$ becomes spread out in time, where the same function prototype $h(x)$ is called basic wavelet. 
IV.3.2 The Comparison of Wavelet Transform and STFT

To compare with the STFT, we choose as a modulated window

$$
h(t)=g(t) e^{-j \omega_{0} t} .
$$

Then the frequency response of the analysis filters indeed satisfy Equation (20) with the identification

$$
s=\frac{\omega}{\omega_{0}} .
$$

But more generally, $h(t)$ can be any band-pass function and the scheme still works.

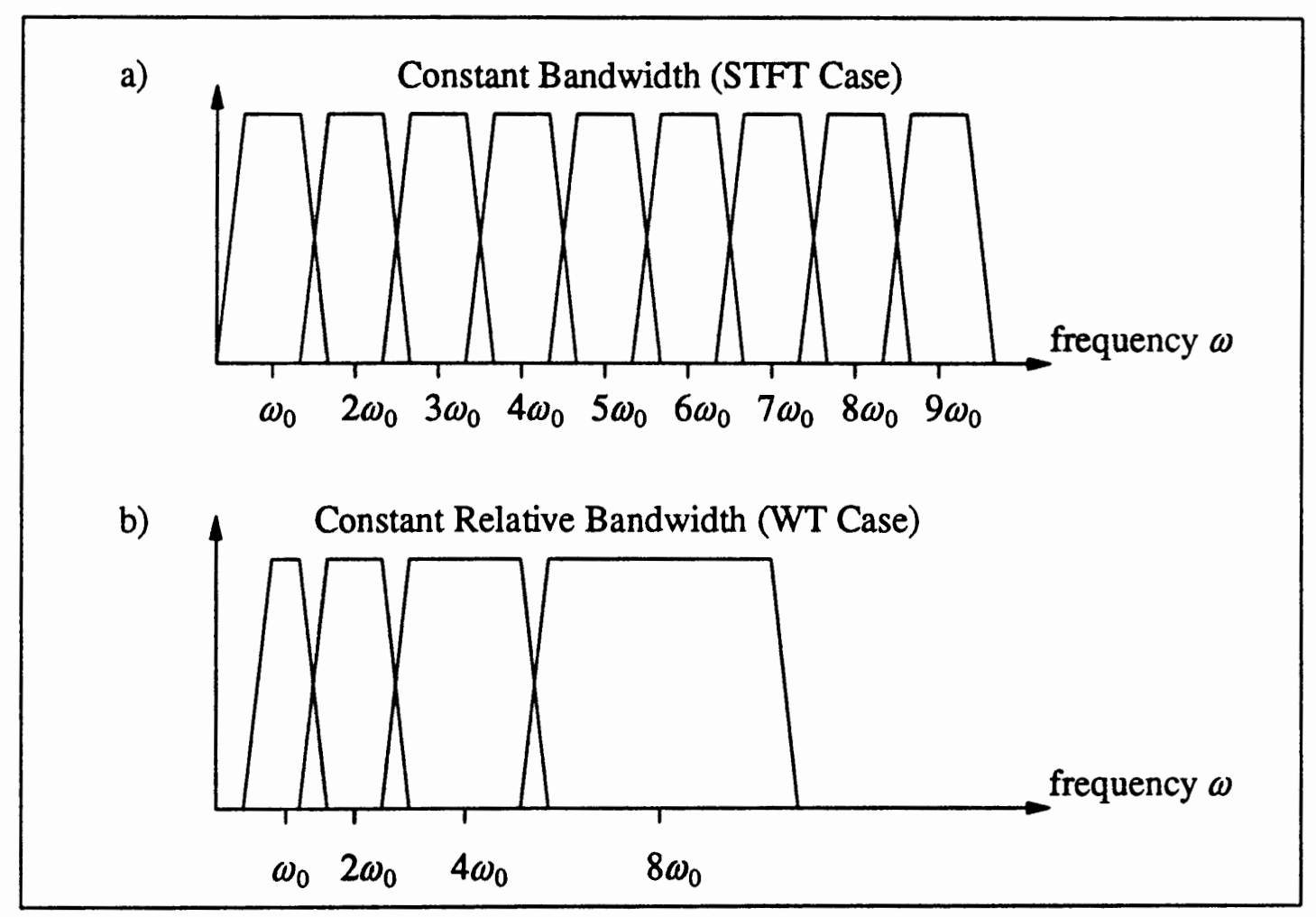

Figure 11. Division of the frequency domain (a) for the STFT (uniform) and (b) for WT (logarithmic coverage). 
It is important to note that here, the local frequency $\omega=s \omega_{0}$ has little to do with that described for the STFT: indeed, it is associated with the scaling scheme. As a result, this local frequency, whose definition depends on the basic wavelet, is no longer linked to frequency modulation (as was the case for the STFT) but is now related to time-scalings. This is the reason why the terminology scale is often preferred to "frequency" for the Continuous wavelet transform, the word frequency being reserved for the STFT. Note that we define scale in wavelet analysis like the scale in geographical maps: since the filter bank impulse responses in Equation (21) are dilated as scale increases, larger scale corresponds to contracted signals, while smaller scale corresponds to dilated signals (see Figure 12).

a)

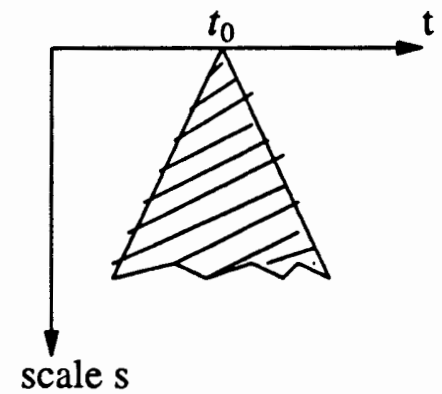

c)

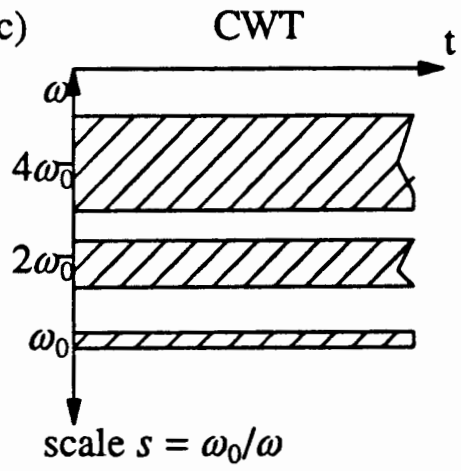

b)

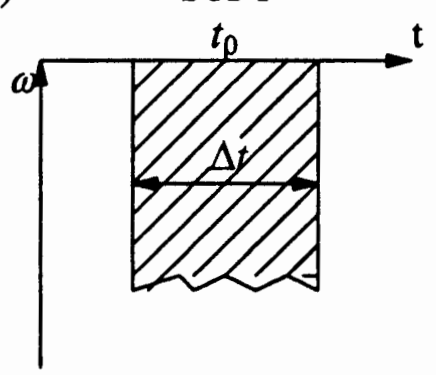

d)

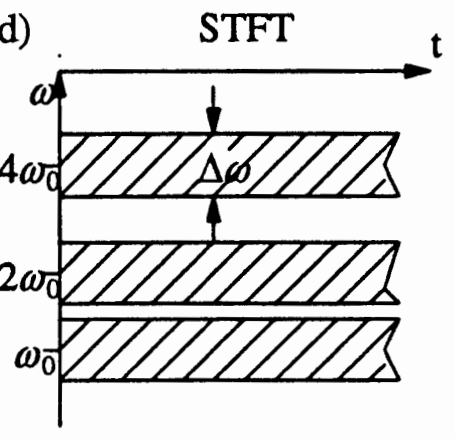

Figure 12. Regions of influence of a Dirac pulse at $t=t_{0}$ for the WT and the STFT 
Figure 12(a) shows that the influence of the signal's behavior around $t=t_{0}$ in the analysis is limited to a cone in the time-scale plane; it is therefore very "localized" around $t_{0}$ for small scales. In the STFT case, the corresponding region of influence is as large as the extent of the analysis window over all frequency, as shown in Figure 12(b). Moreover, since the time-scale analysis is logarithmic in frequency $\omega_{0}$ in the signal increases with $\omega_{0}$ in a scalogram (Figure 12(c)), whereas it remains constant in a spectrogram (Figure 12(d)).

\section{IV.4 AN IMPLEMENTATION OF A PARTICULAR WAVELET TRANSFORM}

\section{IV.4.1 From the Original Definition to A Simple Form}

The definition of wavelet transform, Equation (21), can be seen as inner product of the form

$$
W_{s} f(x)=\int f(t) h_{s, x}(t) d t
$$

which measures the "similarity" between the signal and the basis functions

$$
h_{s, x}=\frac{1}{\sqrt{s}} h\left(\frac{t-x}{s}\right)
$$

or

$$
h_{s}(x)=\frac{1}{\sqrt{s}} h\left(\frac{x}{s}\right)
$$

it is called "wavelets", where $s$ is a scale factor. For $h(x) \in L^{2}$,

$$
\int_{-\infty}^{+\infty}\left|\frac{1}{\sqrt{s}} h\left(\frac{x}{s}\right)\right|^{2} d x=\int_{-\infty}^{+\infty}|h(x)|^{2} d x
$$

so the constant $\frac{1}{\sqrt{s}}$ is used for energy normalization.

In wavelet transform, mostly, the window is chosen to be symmetric, that 
means $h(x)$ is chosen as an even function. So for the even case, after reversing basic wavelet $h(x)$, the wavelet transform can also can be seen as a convolution of a signal and the wavelets. For the reason of calculation simplification the energy normalization constant $\frac{1}{\sqrt{s}}$ is substituted by a sample form $\frac{1}{s}$. This can be considered as function normalization.

Now, the wavelet transform can be written down in a more simple form [9]

$$
W_{s} f(x)=\frac{1}{s} f * \psi\left(\frac{x}{s}\right)
$$

In this thesis, we denote

$$
\xi_{s}(x)=\frac{1}{s} \xi\left(\frac{x}{s}\right),
$$

which is the dilation by a scaling factor $s$ of any function $\xi(x)$. A wavelet transform is computed by convolving the signal with a dilated wavelet. The wavelet transform of $f(x)$ at the scale $s$ and position $x$, computed with respect to the wavelet $\psi(x)$, is defined by

$$
W_{s} f(x)=f * \psi_{s}(x),
$$

with

$$
\psi_{s}(x)=\frac{1}{s} \psi\left(\frac{x}{s}\right) .
$$

This form of wavelet transform is adopted by Mallat in his software package " XWAVE1".

\section{IV.4.2 Dyadic Wavelet Transform}

In this section, we will theoretically discuss how the wavelet transform work. We will focus on the dyadic wavelet transform because for most purposes, the wavelet model is not required to keep a continuous scale parameter $s$. To allow fast numerical 
implementation, we impose that the scale varies only along the dyadic sequence $\left(2^{j}\right)_{j} \in Z[8]$.

If a wavelet is a function $\psi(x)$ whose average is zero, we denote by $\psi_{2}(x)$ the dilation of $\psi(x)$ by a factor $2^{j}$

$$
\psi_{2 j}(x)=\frac{1}{2^{j}} \psi\left(\frac{x}{2^{j}}\right) .
$$

From the definition, the wavelet transform of a function $f(x)$ at the scale $2^{j}$ and at the position $x$ can written as the convolution product

$$
W_{2^{i}} f(x)=f^{*} \psi_{2^{j}}(x) .
$$

It is very easy to know that the Fourier transform of $W_{2 j} f(x)$ is

$$
\hat{W}_{2^{j}} f(\omega)=\hat{f}(\omega) \hat{\psi}\left(2^{j} \omega\right) .
$$

By imposing that there exists two strictly positive constants $A_{1}$ and $B_{1}$ such that

$$
A_{1} \leq \sum_{j=-\infty}^{+\infty}\left|\hat{\psi}\left(2^{j} \omega\right)\right|^{2} \leq B_{1} .
$$

We ensure that the whole frequency axis is covered by dilations of $\hat{\psi}(\omega)$ by $\left(2^{j}\right)_{j \in Z}$ so that $\hat{f}(\omega)$, and thus, $f(x)$ can be recovered from its dyadic wavelet transform. The reconstructing wavelet $\chi(x)$ is any function whose Fourier transform satisfies

$$
\sum_{j=-\infty}^{+\infty} \hat{\psi}\left(2^{j} \omega\right) \hat{\chi}\left(2^{j} \omega\right)=1
$$

If Equation (37) is true, an infinite number of functions $\hat{\chi}(\omega)$ satisfy Equation (38). The function $f(x)$ is recovered from its dyadic wavelet transform with the summation

$$
f(x)=\sum_{j=-\infty}^{+\infty} W_{2^{i}} f * \chi_{2^{j}}(x) \text {. }
$$

This equation is proved by computing its Fourier transform and inserting Equation (36) and Equation (38) a norm equivalence relation 


$$
A_{1}\|f\|^{2} \leq \sum_{j=-\infty}^{+\infty}\left\|W_{2^{j}} f(x)\right\|^{2} \leq B_{1}\|f\|^{2} .
$$

This means that the dyadic wavelet transform is not only complete but also stable. If $B_{1} / A_{1}$ approach to 1 , it will more stable.

A wavelet transform is more than complete; it is redundant. For any sequence $\left(g_{j}(x)\right)_{j \in Z}$, with $g_{j}(x) \in L^{2}$, is not necessarily the dyadic wavelet transform of some function, in $L^{2}$, from Equations (35) and (39), we can have

$$
\sum_{l=-\infty}^{+\infty} g_{l} * K_{l, j}(x)=g_{j}(x)
$$

where

$$
K_{l, j}(x)=\chi_{2^{j}} * \psi_{2 j}(x)
$$

These equations are known as reproducing kernel equations. The energy of the Kernel $K_{l, j}(x)$ measures the redundancy of the wavelet transform at the scales $2^{j}$ and $2^{l}$.

\section{IV.4.3 Discrete Dyadic Wavelet Transform}

In numerical applications, we can not compute the wavelet transform at an arbitrary fine scale. Let us normalize the finest scale to 1 . In order to model this scale limitation, we introduce a real function $\phi(x)$ whose Fourier transform is an aggregation of $\hat{\psi}\left(2^{j} \omega\right)$ and $\hat{\chi}\left(2^{j} \omega\right)$ at scales $2^{j}$ larger than 1

$$
|\hat{\phi}(\omega)|^{2}=\sum_{j=1}^{+\infty} \hat{\psi}\left(2^{j} \omega\right) \hat{\chi}\left(2^{j} \omega\right) .
$$

We suppose that here $\hat{\psi}(\omega) \hat{\chi}(\omega)$ is a real, positive even function. From Equation (38), one can prove that the integral of $\phi(x)$ is equal to 1 and, hence, that it is a smoothing function. We denote

$$
S_{2^{j}} f(x)=f * \phi_{2^{j}}(x) \text { with } \phi_{2^{j}}(x)=\frac{1}{2^{j}} \phi\left(\frac{x}{2^{j}}\right) .
$$


If the scale $2^{j}$ is larger, the more details of $f(x)$ are removed by $S_{2^{j}}$. For any scale $2^{J}>1,(43)$ yields

$$
|\hat{\phi}(\omega)|^{2}-\left|\hat{\phi}\left(2^{J} \omega\right)\right|^{2}=\sum_{j=1}^{J} \hat{\psi}\left(2^{j} \omega\right) \hat{\chi}\left(2^{j} \omega\right)
$$

We can also derive, from this equation, that the higher frequencies of $S_{1} f(x)$ have disappeared in $S_{2^{J}} f(x)$.

We suppose that the original signal is a discrete sequence $D=\left(d_{n}\right)_{n} \in Z$ of finite energy. If there exists two constants $C_{1}>0$ and $C_{2}>0$ such that $\hat{\phi}(\omega)$ satisfies

$$
C_{1} \leq \sum_{n=-\infty}^{+\infty}|\hat{\phi}(\omega+2 n \pi)|^{2}<C_{2}
$$

than we can prove Equation (41) that there exists a function $f(x) \in L^{2}$ such that

$$
S_{1} f(x)=d_{n}
$$

The input signal can thus rewritten $D=\left(S_{1} f(n)\right)_{n \in Z}$. Let us denote

$$
W_{2^{j}}^{d} f=\left(W_{2^{j}} f(n+\omega)\right)_{n \in Z} \text { and } S_{2^{j}}^{d} f=\left(S_{2^{j}} f(n+\omega)\right)_{n \in Z}
$$

where $\omega$ is a sampling shift and depends only on $\psi(x)$. For any coarse scale $2^{J}$, the sequence of discrete signals

$$
S_{2^{j}}^{d} f,\left(W_{2^{j}}^{d} f\right)_{1 \leq j \leq J}
$$

is called the discrete wavelet transform of $D=\left(S_{1} f(n)\right)_{n \in Z}$.

IV.4.4 Fast implementation of discrete algorithms.

We impose that the Fourier transform of the smoothing function $\phi(x)$ defined by Equation (43) can be written as an infinite product

$$
\hat{\phi}(\omega)=e^{-j \omega \omega} \prod_{p=1}^{+\infty} H\left(2^{-p \omega}\right)
$$

where $H(\omega)$ is a $2 \pi$ periodic differentiable function such that 


$$
|H(\omega)|^{2}+|H(\omega+\pi)|^{2} \leq 1 \text { and }|H(0)|=1
$$

We can prove Equation (40) that the conditions in Equation (50) are sufficient so that Equation (49) defines a smoothing function $\phi(x)$, which is in $L^{2}$. The parameter $w$ is the sampling shift that was introduced in the last section. It is adjusted in order so that $\phi(x)$ is symmetrical with respect to 0 . Equation (49) implies that

$$
\hat{\phi}(2 \omega)=e^{-j w \omega} H(\omega) \hat{\phi}(\omega) .
$$

We define a wavelet $\psi(x)$ whose Fourier transform $\hat{\psi}(\omega)$ is given by

$$
\hat{\psi}(2 \omega)=e^{-j \omega \omega} G(\omega) \hat{\phi}(\omega)
$$

where $G(\omega)$ is a $2 \pi$ periodic function. Equation (45) for $J=1$ proves that $\hat{\psi}(\omega), \hat{\chi}(\omega)$, and $\hat{\phi}(\omega)$ must satisfy

$$
\hat{\psi}(2 \omega) \hat{\chi}(2 \omega)=|\hat{\phi}(\omega)|^{2}-|\hat{\phi}(2 \omega)|^{2} .
$$

Let us impose that $\hat{\chi}(\omega)$ can be written

$$
\hat{\chi}(2 \omega)=e^{i w \omega} K(\omega) \hat{\phi}(\omega)
$$

If we insert Equations (51) and (54) into Equation (53), we obtain

$$
|H(\omega)|^{2}+G(\omega) K(\omega)=1 .
$$

One can prove that Equation (55) is sufficient to define $K(\omega)$ such that $\hat{\chi}(\omega)$ is the Fourier transform of a reconstructing wavelet that satisfies Equation (38).

We want a wavelet $\phi(x)$ equal to first-order derivative of a smoothing function $\theta(x)$. This implies that $\hat{\psi}(\omega)$ must have a zero of order 1 at $\omega=0$. Since $|\hat{\phi}(0)|=1$, Equation (52) yields that $G(\omega)$ must have a zero of order 1 at $\omega=0$. We choose $H(\omega)$ in order to obtain a wavelet $\psi(x)$, which is antisymmetrical, as regular as possible, and has a small compact support. A family of $2 \pi$ periodic functions $H(\omega), G(\omega)$, and $K(\omega)$ that satisfy these constraints is given by 


$$
\begin{aligned}
& H(\omega)=e^{i \omega / 2}(\cos (\omega / 2))^{2 n+1}, \\
& G(\omega)=4 i e^{i \omega / 2} \sin (\omega / 2), \\
& K(\omega)=\frac{1-|H(\omega)|^{2}}{G(\omega)} .
\end{aligned}
$$

The $2 \pi$ periodic function $H(\omega), G(\omega)$, and $K(\omega)$ can be as the transfer function discrete filters with From Equations (49) and (52), we derive

$$
\begin{aligned}
& \hat{\phi}(\omega)=\left(\frac{\sin (\omega / 2)}{\omega / 2}\right)^{2 n+1}, \\
& \hat{\psi}(\omega)=i \omega\left(\frac{\sin (\omega / 4)}{\omega / 4}\right)^{2 n+2} .
\end{aligned}
$$

The Fourier transform $\hat{\theta}(\omega)$ of the primitive is therefore

$$
\hat{\theta}(\omega)=\left(\frac{\sin (\omega / 4)}{\omega / 4}\right)^{2 n+2} .
$$

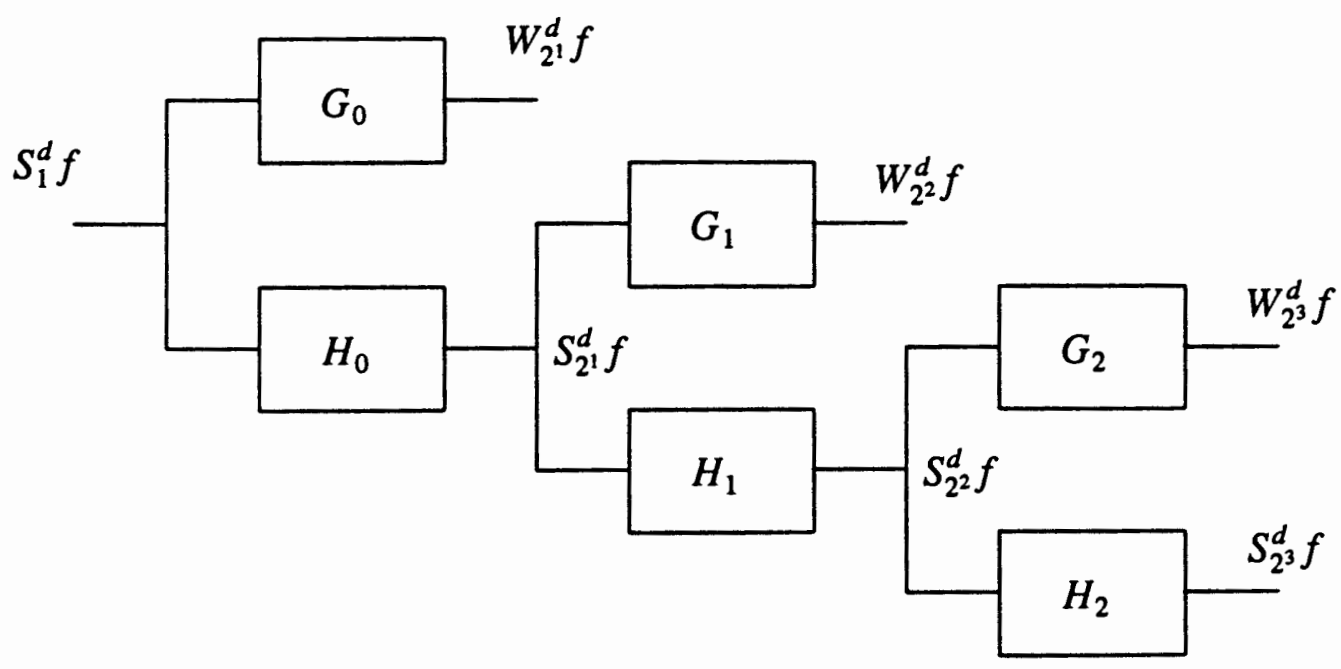

Figure 13. Block diagram of the discrete wavelet transform implemented with discrete-time filters.

The $2 \pi$ periodic function $H(\omega), G(\omega)$, and $K(\omega)$ can be as the transfer function discrete filters with finite impulse. 
We denote $H_{p}, G_{p}$, and $K_{p}$ the discrete filters obtained by putting $2^{p}-1$ zeros between each of the coefficients of the filters $H, G$, and $K$. The transfer function of these filters is, respectively, $H\left(2^{p} \omega\right), G\left(2^{p} \omega\right)$, and $K\left(2^{P} \omega\right)$.

The following algorithm computes the discrete wavelet transform of the discrete signal $S_{1}^{d} f$, At each scale $2^{j}$, it decomposes $S_{2^{j}}^{d} f$ into $S_{2^{j}}+1^{d} f$ and $W_{2^{j}}+1^{d} f$.

$j=0$

while $(j<J)$,

$$
\begin{aligned}
& W_{2^{j+1}}^{d} f=\frac{1}{\lambda_{j}} \cdot S_{2 j}^{d} f * G_{j} \\
& S_{2^{j+1}}^{d} f=S_{2 j}^{d} f * H_{j} \\
& j=j+1
\end{aligned}
$$

end of while.

At each scale $2^{j}$, we divide the values displayed in the sample set of $S_{2^{j}}^{j} f * G_{j}$ by $\lambda$ to obtain accurate measures of Lipschitz exponents from the wavelet maxima. Due to discretization, the wavelet modulus maxima of a step edge do not have the same amplitude at all scales as they should in a continuous model. The constants $\lambda$ compensate for this discrete effect. The inverse wavelet transform algorithm reconstructs $S_{1}^{d} f$ from the discrete dyadic wavelet transform. At each scale $2^{j}$, it reconstructs $S_{2^{j-1}}^{d} f$ from $S_{2^{j}}^{d} f$ and $W_{2^{j}}^{d} f$.

$j=J$

while $(j>0)$

$$
\begin{aligned}
& S_{2 j-1}^{d} f=W_{2 j}^{d} f * \tilde{G}_{j-1}+S_{2 j}^{d} f * \tilde{H}_{j-1}, \\
& j=j-1,
\end{aligned}
$$


end of while.

\section{IV.5 SPLINE WAVELET TRANSFORM}

\section{IV.5.1 Smoothing Function}

We call a smoothing function any function $\theta(x)$ whose integral is equal to 1 and that converges to 0 at infinity. So

$$
\int_{-\infty}^{+\infty} \theta(x) d x=1
$$

and

$$
\begin{aligned}
& \lim _{x \rightarrow+\infty} \theta(x)=0 \\
& \lim _{x \rightarrow-\infty} \theta(x)=0 .
\end{aligned}
$$

We assuming that $\theta(x)$ is once differentiable, $\psi^{1}(x)$ is the first derivative of $\theta(x)$

$$
\psi^{1}(x)=\frac{d \theta(x)}{d x}
$$

By definition, the function $\psi^{1}(x)$ can be considered to be wavelet because its integral is equal to 0 .

$$
\int_{-\infty}^{+\infty} \psi^{1}(x) d x=0
$$

In this thesis, the spline functions $\beta^{n}(x)$ are chosen to be the smoothing functions $\theta(x)$.

The function $\beta^{n}(x)$ can be generated by repeated convolutions of zero order splines [13]

$$
\beta^{n}(x)=\beta^{0}(x) * \beta^{n-1}(x)
$$

where $\beta^{0}(x)$ is the indicator function in the interval $[-1 / 2,1 / 2)$, as shown in Fig. 15(a). 
First-order B-spline function $\beta^{1}(x)$, quadratic spline function $\beta^{2}(x)$ and cubic spline function $\beta^{3}(x)$ that can be obtained by Equation (67) are shown in Figure 15(b),(c) and (d). The spline wavelets $\psi^{n}(x)$ that are related to spline function $\beta^{n}(x)$ are shown in Figure 15.

From Figure 14, we can observe that the spline functions smooth themselves in keeping with the convolving of its 0-order function. So when other signals, e.g. EEG signals, convolve with smoothing functions, spline functions, they are also smoothed.

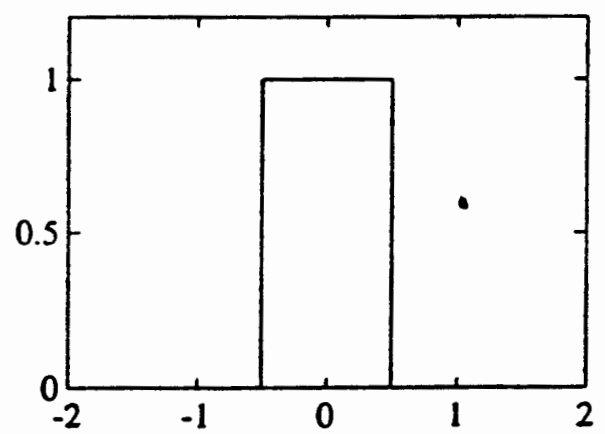

(a)

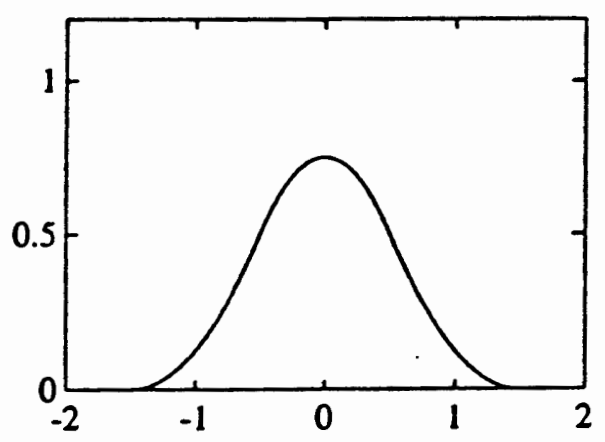

(c)

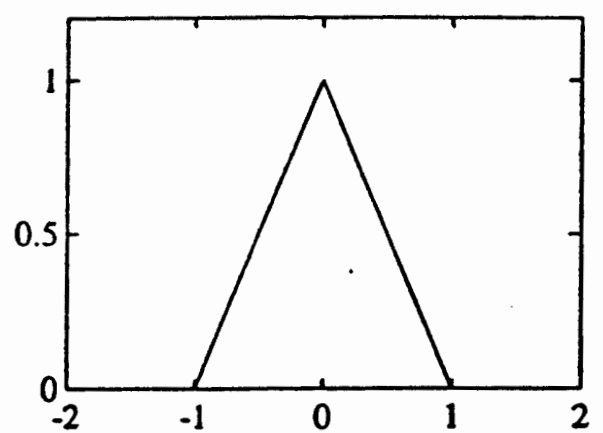

(b)

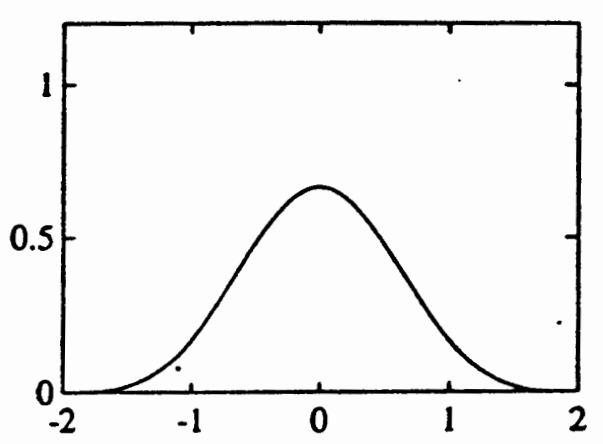

(d)

Figure 14. The family of Spline function. 
For our ERP's waveform analysis in this thesis, we choose the third-order spline, cubic spline, to be the smoothing function and its first-order derivative, quadratic spline, to be the wavelet.
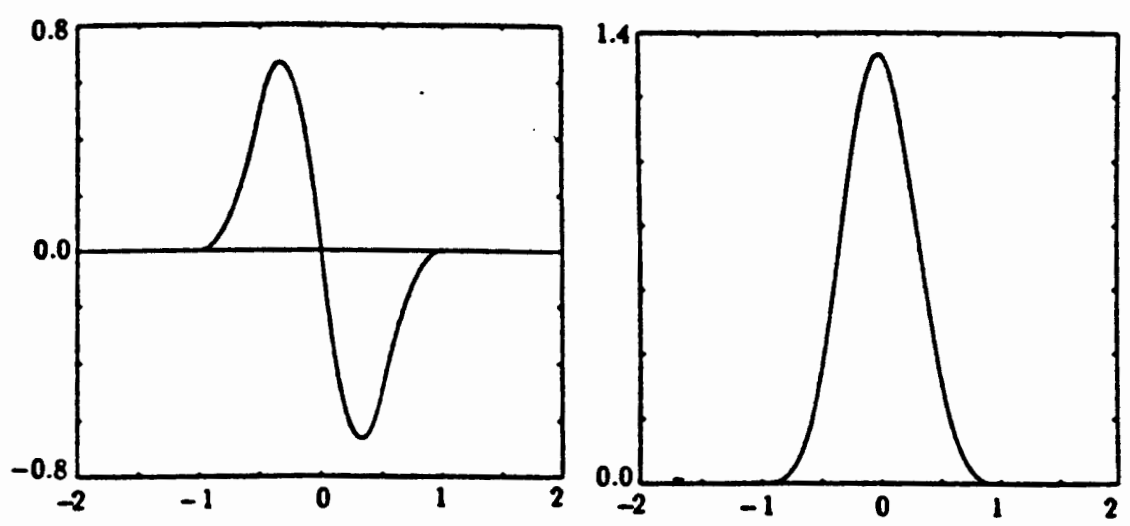

Figure 15. Figure (a) is a quadratic spline wavelet of compact support. It is the derivative of the smoothing function $\theta(x)$ shown in (b).

From Equation (67), we know that the cubic spline is the result of four 0-order splines convolving together. We can write

$$
\theta(x)=\beta^{0}(x) * \beta^{0}(x) * \beta^{0}(x) * \beta^{0}(x)
$$

For one single 0-order spline, which is a rectangular function, its Fourier transform

$$
\hat{\beta}^{0}(\omega)=\frac{\sin (\omega / 4)}{\omega / 4}
$$

So the cubic spline's Fourier transform

$$
\hat{\theta}(\omega)=\left(\frac{\sin (\omega / 4)}{\omega / 4}\right)^{4}
$$

It is the same with (61) when $n=2$. We also can obtain the Fourier transform of its 
first-order derivative, quadratic spline wavelet function

$$
\hat{\psi}(\omega)=i \omega\left(\frac{\sin (\omega / 4)}{\omega / 4}\right)^{4} .
$$

\section{IV.5.2 Local Maxima of Spline Wavelet Transform}

Recall Equation (37),

$$
\psi^{1}(x)=\frac{d \theta(x)}{d x}
$$

and

$$
\psi_{s}^{1}(x)=\frac{1}{s} \psi^{1}\left(\frac{x}{s}\right)
$$

So we can derive

$$
\psi_{s}^{1}=\frac{1}{s} \frac{d \theta\left(\frac{x}{s}\right)}{d\left(\frac{x}{s}\right)}=\frac{d \theta_{s}(x)}{d\left(\frac{x}{s}\right)}=s \frac{d \theta_{s}\left(\frac{x}{s}\right)}{d x}
$$

where

$$
\theta_{s}(x)=\frac{1}{s} \theta\left(\frac{x}{s}\right)
$$

From (31) and (33), we can derive that

$$
W_{s}(x)=f *\left(s \frac{d \theta_{s}}{d x}\right)(x)=s \frac{d}{d x}\left(f * \theta_{s}\right)(x)
$$

So, we can see that the wavelet transform $W_{s} f(x)$ is proportional to the first order derivative of the signal smoothed by $\theta_{s}(x)$. For a fixed scale $s$, the local extrema of $W f(s, x)$ along the $x$ variable, correspond to the inflection points of $f * \theta_{s}(x)$. An inflection point of $f * \theta_{s}(x)$ can either be a maximum or a minimum of its first derivative. Like at the abscissa $x_{0}$ and $x_{2}$ of Figure 10, the local maxima of the first derivative are sharp ascending variation points of $f * \theta_{s}(x)$, which correspond to the 


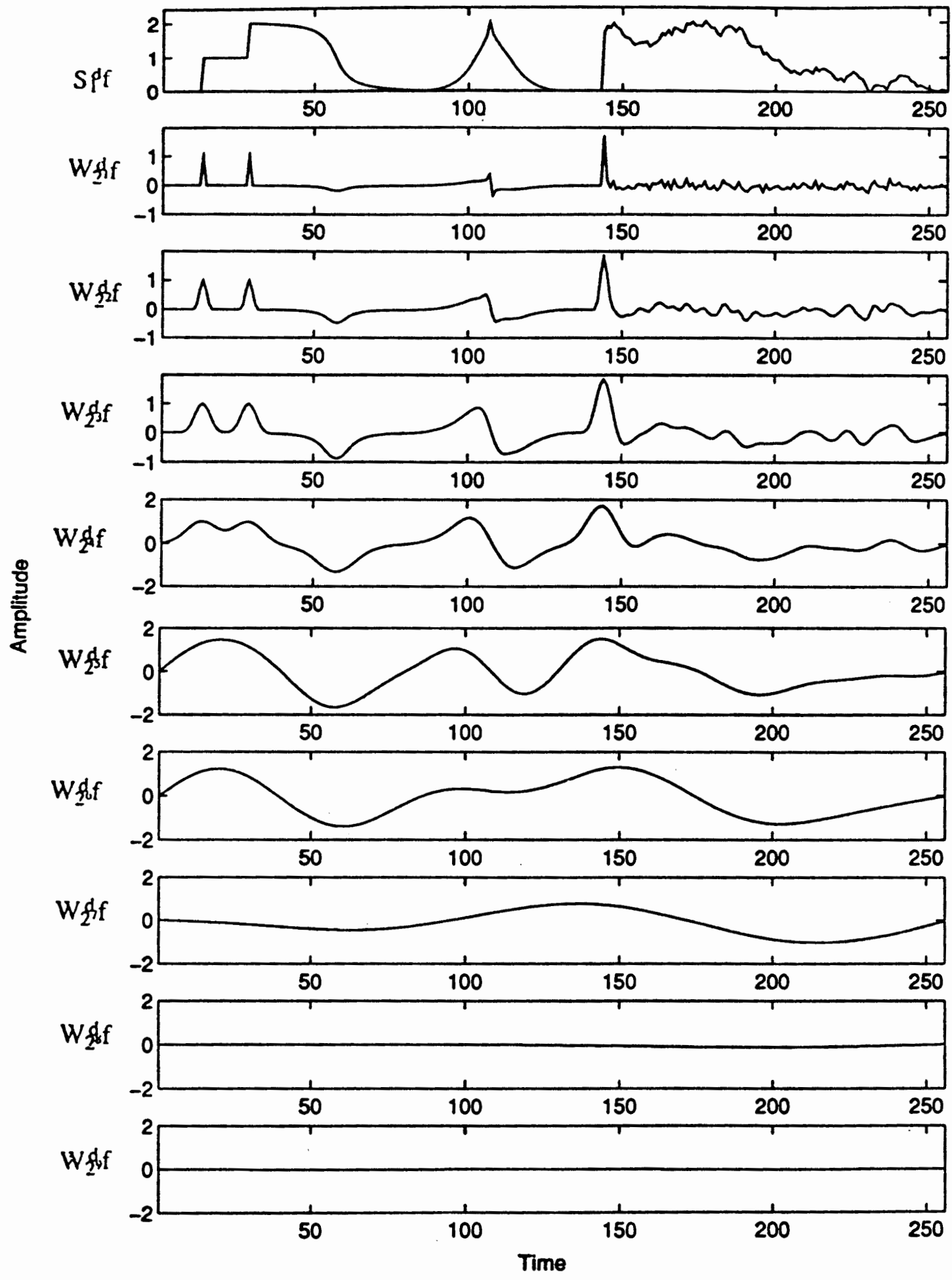

Figure 16. Signal of 256 sample and its dyadic wavelet transform. 


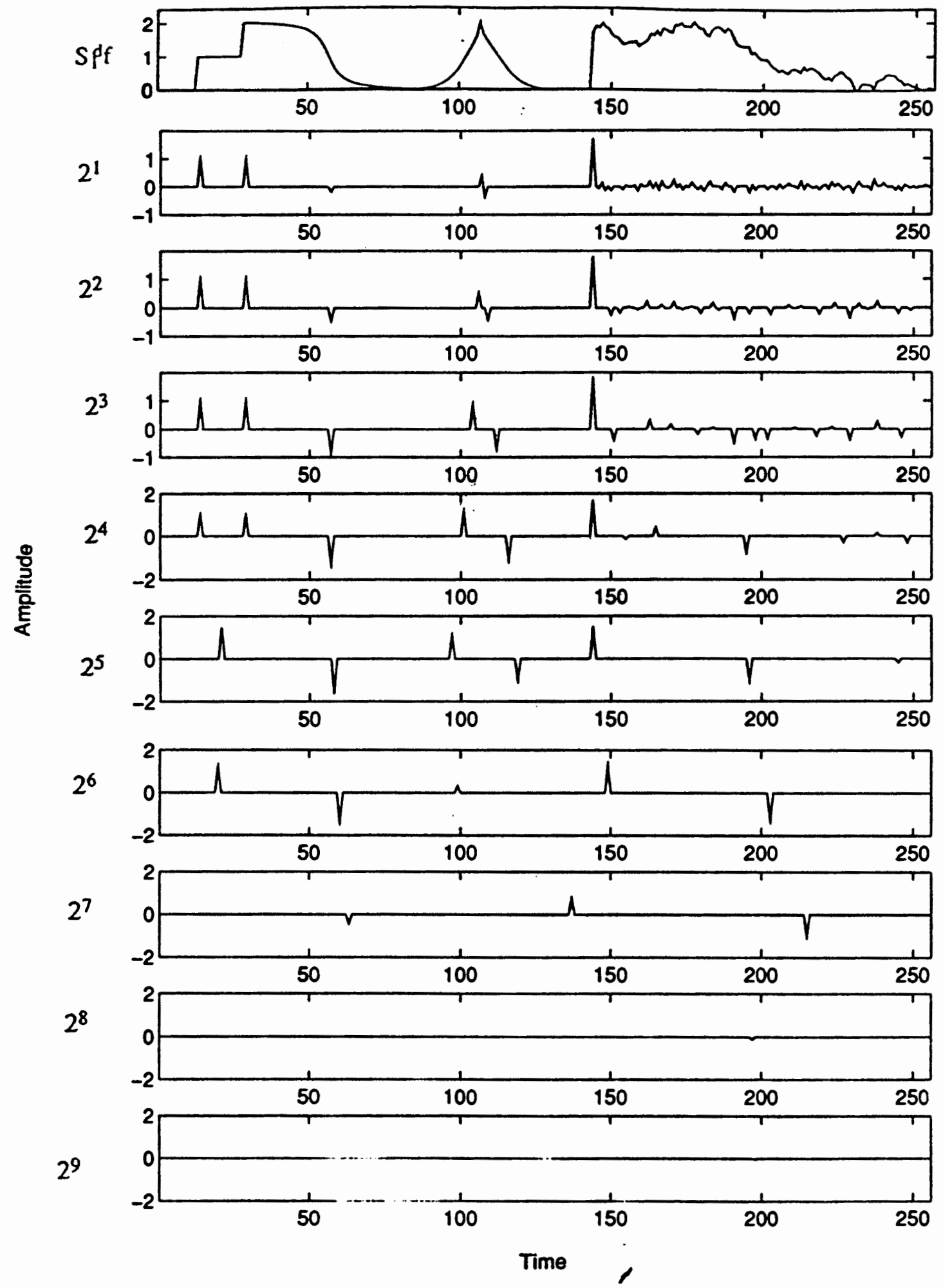

Figure 17. Signal of 256 sample and its local maxima of the dyadic WT of signal show in Figure 16. 
fast discrease points. These two types of sharp variation points can be distinguished by observing whether an extremum of $W f(s, x)$ is a maximum or a minimum. For this thesis, we are also interested in the location of the local maxima of $|W f(s, x)|$.

In practice, the original discrete signal $D$ has a finite number $N$ of nonzero values: $D=\left(d_{n}\right)_{1 \leq n \leq N}$. To solve the border problems, we use the same periodization technique as in a cosine transform. we suppose that our signal has a period of $2 \mathrm{~N}$ samples, and we extend it with a symmetry for $N<n \leq 2 N: d_{n}=d_{2 N+1-n}$. By periodizing the signal with a symmetry, we avoid creating a discon

From the discrete wavelet transform, at each scale $2^{j}$, we detect the modulus maxima by finding the points where $\left|W_{2^{j}} f(n+\omega)\right|$ is larger than its two closest neighbor values and strictly larger than at least one of them. Functions are respectively $\overline{H(2 j \omega)}$ and $\overline{G(2 j \omega)}$ (complex conjugates of $H(2 j \omega)$ and $G(2 j \omega)$ ).

Watelet transform, at nine scales, of a sample signal provided by XWAVE1 are shown in Figure 16 and Figure 17 are the corresponding local maxima.

\section{IV.5.3 Local Maxima of WT and Regularity of Signal}

Signal sharp variations produce modulus maxima at different scales $2^{j}$. We know that the value of a modulus maximum at scale $2^{j}$ measures the derivative of the signal smoothed at scale $2^{j}$, but it is not clear how to combine these different values to characterize the signal variation [10]. The wavelet theory gives an answer to this question by showing that the evolution across scales of wavelet transform depends on the local Lipschitz regularity of the signal [11].

Definition: Let $0 \leq \alpha \leq 1$. A function $f(x)$ is uniformly Lipschitz $\alpha$ over an interval $[\mathrm{a}, \mathrm{b}]$ if and if there exits a constant $\mathrm{K}$ such that for any $\left(x_{0}, x_{1}\right) \in[\mathrm{a}, \mathrm{b}]$

$$
\left|f\left(x_{0}\right)-f\left(x_{1}\right)\right| \leq K\left|x_{0}-x_{1}\right|^{\alpha} .
$$

We refer to the Lipschitz uniform regularity of $f(x)$ as the upper bound $\alpha_{0}$ of all $\alpha$ 
such that $f(x)$ is uniformly Lipschitz $\alpha$.

If $f(x)$ is differentiable at $x_{0}$, then it is Lipschitz $\alpha=1$. If the uniform Lipschitz regularity $\alpha_{0}$ is larger, the singularity at $x_{0}$ will be more "regular". If $\mathrm{f}(\mathrm{x})$ is discontinuous but bounded in the neighborhood of $x_{0}$, its uniform Lipschitz regularity in the neighborhood of $x_{0}$ is 0 . The following Theorem proves that the Lipschitz exponent of a function can be measured from the evolution across scales of the absolute value of the wavelet transform.

Theorem: Let $0<\alpha<1$. A function $f(x)$ is uniformly Lipschitz $\alpha$ over [a,b] if and only if there exists a constant $K>0$ such that for all $x \in[\mathrm{a}, \mathrm{b}]$, the wavelet transform satisfies

$$
\left|W_{2 j} f(x)\right| \leq K(2 j)^{\alpha}
$$

So that wavelet transform can not only detect sharp variations but also characterize the local regularity of signal. The proof of this theorem can be found in [11].

We showed that short-time Fourier transform and wavelet transform represent alternative ways to divide the time-frequency (or time-scale) plane. In contrast to STFT, which uses a single analysis window, the WT uses shorter windows at higher frequencies and longer windows at lower frequencies. For some particular wavelet functions, the local maxima of the wavelet transform correspond to the sharp variation points of the signal [12]. When detecting locations of local maxima, we can also record the values of $\mathrm{W}_{\mathbf{s}} \mathrm{f}(\mathrm{x})$ at these locations, which measure the derivative at inflection points. The fast wavelet algorithm and a family of Spline wavelets were also discussed. Because of Spline's compact support, it has many applications. In our study, we used quadratic Spline wavelet as a basic wavelet.

\section{IV.5.4 Daubechies Wavelets---Another Approch for ERP Analysis}


Daubechies wavelets are scaling functions. It can decompose a signal into a set of orthogonal time domain signals for different frequency bands and has a perfect reconstruction property. We know that the frequencies of EEG waves range from 1 to $30 \mathrm{~Hz}$ and have been divided for interpretation purposes into four standard band and the 5 orthogonal Daubechies wavelet decomposed series correspond roughly to frequency bands $(0-4 \mathrm{~Hz}),(4-8 \mathrm{~Hz}),(8-16 \mathrm{~Hz})$ and $(32-64 \mathrm{~Hz})$. They are very close to the four standard bands, Delta, Theta, Alpha and Beta, respectively.

\section{IV.6 EEG SIGNAL PROCESSING USING SPLINE WAVELET TRANSFORM}

\section{IV.6.1 Scale Versus Frequency}

As mentioned in Chapter II, EEG waves range from $1-30 \mathrm{~Hz}$ and often the EEG includes some degree of high frequency $(60 \mathrm{~Hz})$ electrotic noise. A number of studies have observed that $P_{300}$ waveform which is related to the memory processes has the frequencies from $2-4 \mathrm{~Hz}$. Spline wavelet transform first cuts off the higher frequencies by smoothing signals at different scales and then detects the slope of the signal. So wavelet transform only detects the larger structure when it is in larger scales that versus the lower frequencies. But if the scale is too large, some detail information will be lost. In our study, we want to choose a certain scale which can make the transform cut off the frequencies higher than $4 \mathrm{~Hz}$.

The length of the basic spline wavelet in XWAVE1 is 2 points. Its corresponding smoothing function is also 2 points in length (shown in Figure 15). We use 256 sampling points for one single trail or sweep, representing 1024 milliseconds. For $4 \mathrm{~Hz}$ or lower frequencies components, they can complete approximately 4 or fewer periods within this 256 point-period. So we need to choose the window length of wavelet transform one fourth of 256 points. It is 64 points in length. It is easy to know that the window dilates to be 64 points in length when $s=32=2^{5}$. 
We build a approximate $\delta$ function, giving a very large value, e.g. 1000 in one point and padding zeros in both sides of this point. Because of the property of $\delta$ function,

$$
\delta * f(x)=f(x)
$$

We know that the wavelet transforms, at different scales, of $\delta$ function should just be the wavelets themselves. Figure 18. shows that one at scale $2^{5}$. Its actual length is 62 points instead of the theoretic 64 points because the $\delta$ function which we are using here is not a perfect one.
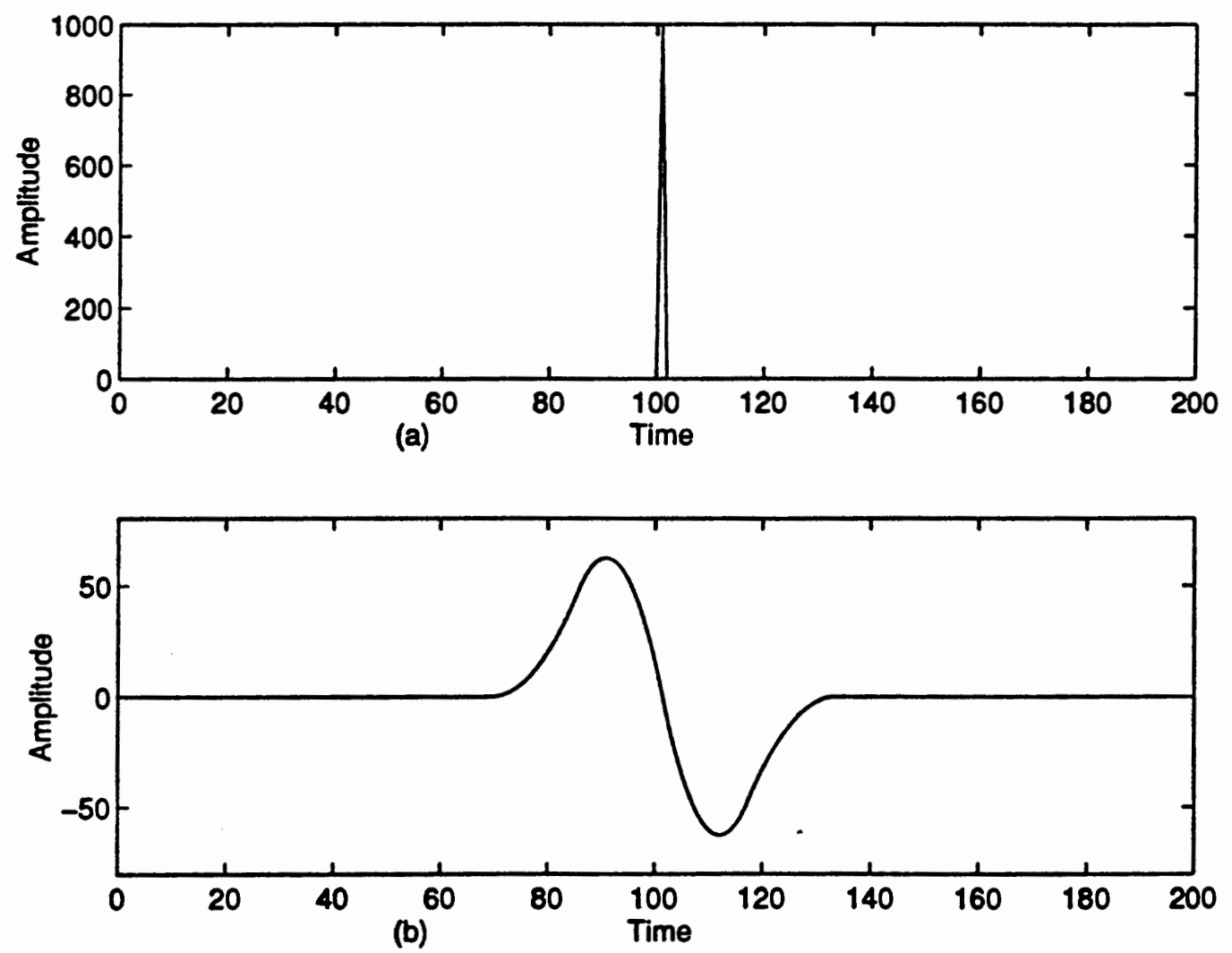

Figure 18. (a) is a approximate $\delta$ function, and (b) is its wavelet transform at scale $2^{5}$. 
We want to use the $2^{5}$ scale spline wavelet transform to detect the slopes of P300 waveforms and the local maxima to measure the sharp variation points in the waveform.

\section{IV.6.2 ERP's Sharp Variation Point Detection}

Electrophysiologic investigations have suggested that the sharpest ascending variation point in a $P_{300}$ wave corresponds to the period that new neural networks are being most rapidly recruited, and the sharpest descending variation point corresponds to the time that these neurals are most rapidly resuming their resting state.

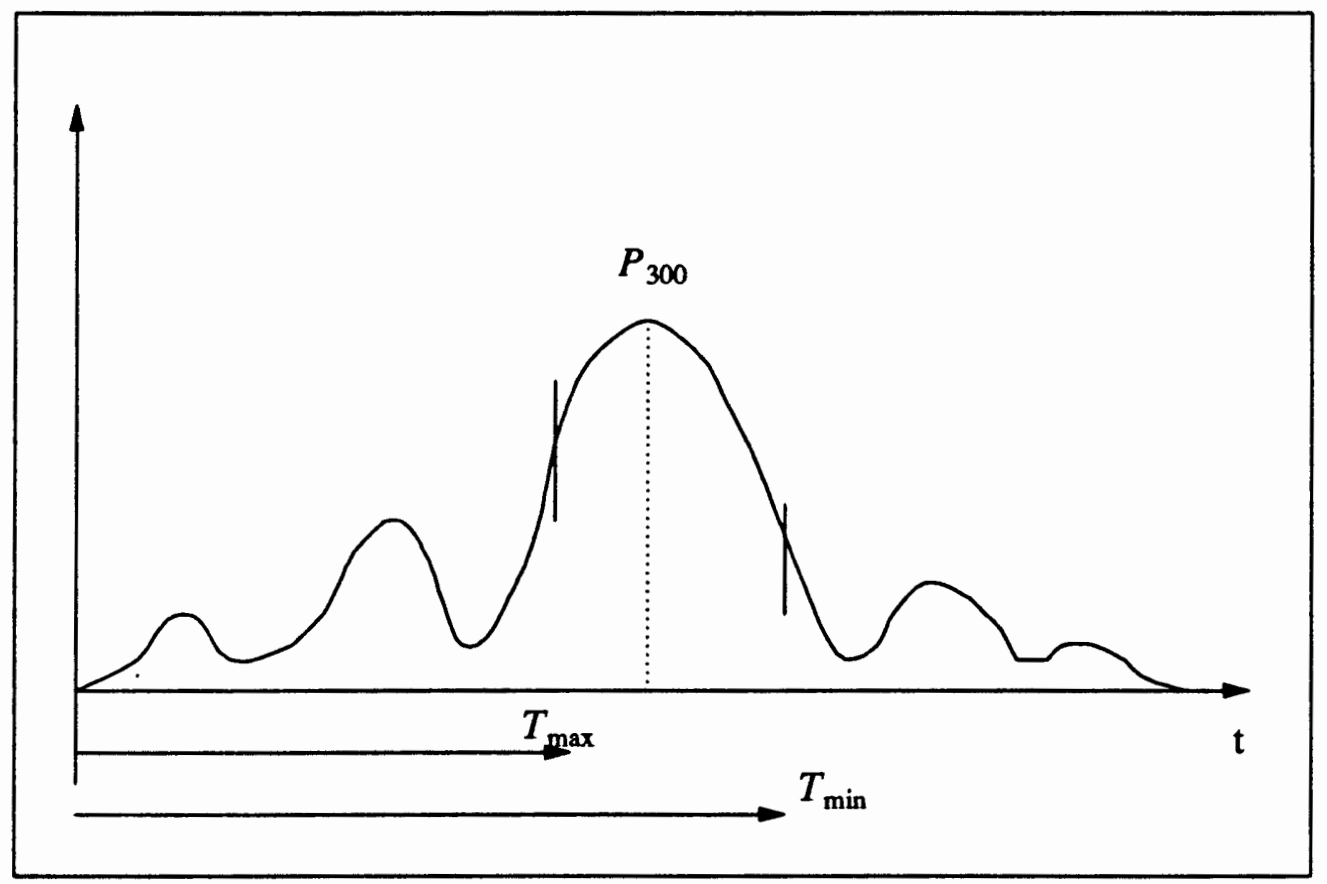

Figure 19. The sharpest variation point of a latency.

Some of the activity underlying the $P_{300}$ and late positive complex (LPC) appear to relate to encoding, or memory processes. To a stimulus, normal subjects have a relatively rapid and steep onset of the $P_{300} /$ LPC. On the contrary, memoryimpaired subjects would show delayed and more slowly rising $P_{300} / \mathrm{LPC}$ components. So both of the slope values and their time locations are of interest in our research. 
1. Decompose the 160 sweeps of EEG signal at scale $2^{5}$ and find the local maxima of its wavelet transform.

2. The same procedure used in Chapter III is repeated here to find the maximum value and minimum value in each sweep of the local maxima. Meanwhile, their time locations are recorded for condition 2 or condition 3 respectively.

3. Substract the maximum value of either condition 2 or condition 3 with the maximum value of the first condition 1 . Count the percentage that the substraction results are greater than zero for condition 2 and condition 3 respectively.

4. The big slopes caused by the eyeblinks which were detected in the matched filtering are removed.

We are also interested in the durations between the positive sharp slopes and the negative ones. Some electrophysiologists theorize that intact subjects should have an earlier-onset and longer-duration $P_{300} /$ PLC than memory-impaired people. Comparing the waveform of Figure 3. with the one of Figure 4, we can find the normal person's waveform has longer and larger late positive complex but the memoryimpaired person's waveform has relatively lower and shorter late positive complex.

For each effective target word, we measure the time, $t_{\max }$, when the sharpest ascending variation point appears and the time, $t_{\min }$, when the sharpest descending variation point appears, respectively, using the reference time, $t_{0}$, at the beginning of the stimulus.

From Table 2. and Table 3., we did not find a consistent pattern between normal and abnormal subjects. The main reason is the EEG signal's very low SNR (< $0 \mathrm{~dB})$. It might cause the wavelet pick up the slope at wrong place. We will discuss more in Chapter V. 

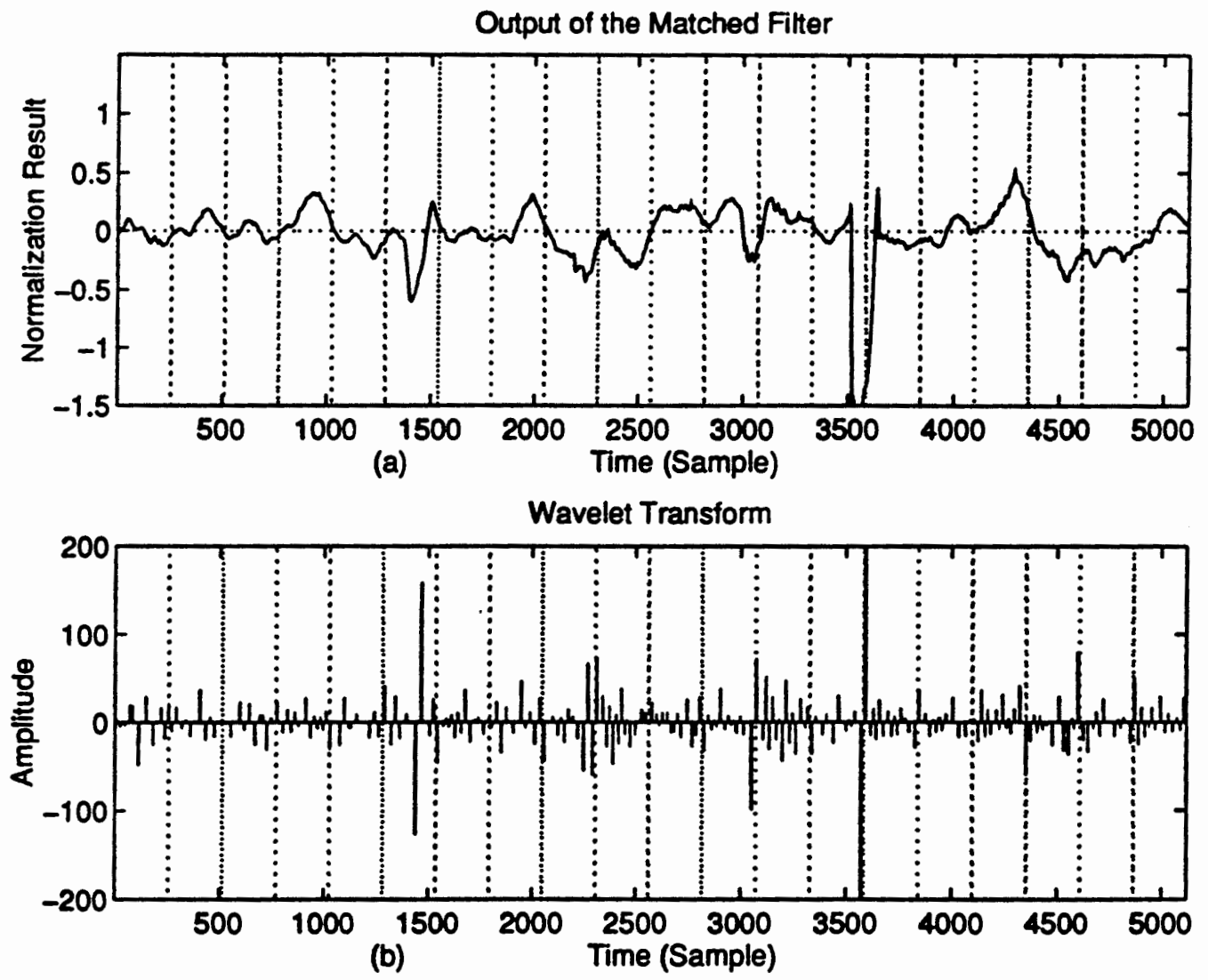

Figure 20. local maxima of the wavelet transform of EEG signal at scale $2^{5}$. 


\begin{tabular}{|c|c|c|c|c|}
\hline Group & Subject & Trail & Conditom $2(\%)$ & Condition $3(\%)$ \\
\hline \multirow{9}{*}{ Normal } & 01 & I & 44 & 57 \\
\hline & & II & 33 & 28 \\
\hline & 02 & I & 40 & 56 \\
\hline & & II & 58 & 69 \\
\hline & 03 & I & 57 & 25 \\
\hline & & II & 28 & 33 \\
\hline & 04 & $I$ & 50 & 17 \\
\hline & & II & 37 & 42 \\
\hline & 05 & I & 52 & 46 \\
\hline \multirow{10}{*}{ Impaired } & 06 & I & 31 & 27 \\
\hline & & II & 68 & 55 \\
\hline & 07 & I & 55 & 58 \\
\hline & & II & 55 & 57 \\
\hline & 08 & I & - & - \\
\hline & & II & 47 & 85 \\
\hline & 09 & I & 30 & 27 \\
\hline & & II & 41 & 60 \\
\hline & 10 & I & 46 & 38 \\
\hline & 11 & $I$ & 45 & 50 \\
\hline
\end{tabular}

Table 2. The ERP analysis result using wavelet transform. 


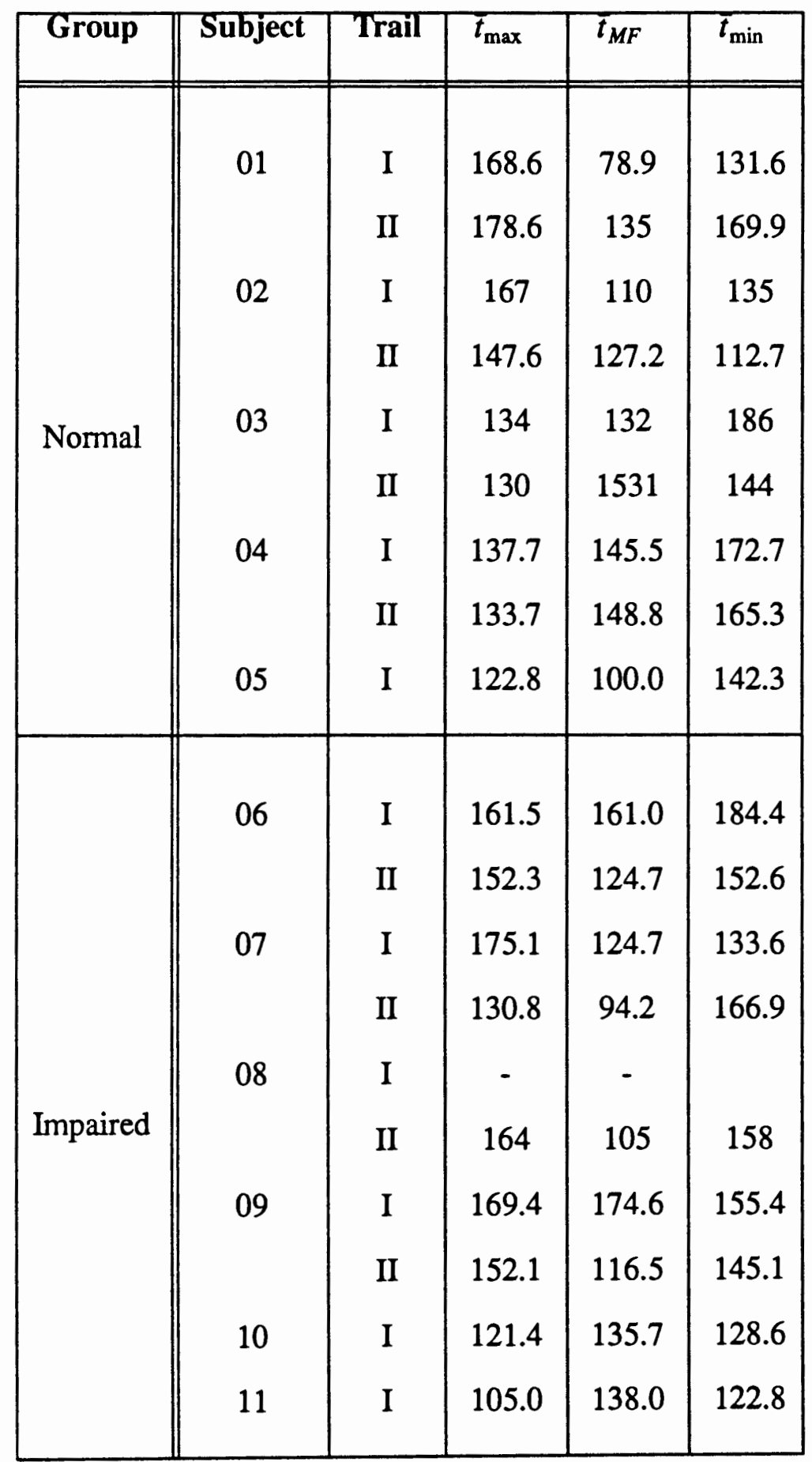

Table 3. The means of time points. $\bar{t}_{\max }$ and $\bar{t}_{\min }$ for wavelet transform's sharpest variation points and $\bar{t}_{M F}$ is the mean time that peaks appear in the matched filter. 


\section{CHAPTER V}

\section{A COMPARISON OF THE WAVELET WITH MATCHED FILTER}

\section{V.1 INTRODUCTION}

Computation of the correlation coefficients is a convenient indicator of how dependent two random variables are on each other. In a memory process, the peak of the response measures how deep or how strong the subjest response is and the sharpest variation point is related to the most exciting moment of the response. we will calculate the correlation coefficients between the peak values of the matched filter output function and the sharpest slopes. We are also interested in the relationship between their time latencies.

In the last two chapters, we processed the EEG signals using the matched filter and wavelet transform. However, the ERP analysis did not show the same result. We will try to analyze the reason which caused the differences in the result.

\section{V.2 ERP ANALYSIS USING CORRELATION COEFFICIENTS}

\section{V.2.1 Correlation Coefficients}

For two random variables, $\mathrm{X}$ and $\mathrm{Y}$, the correlation coefficient between them is defined as

$$
\rho=\frac{\mu}{\sigma_{X} \sigma_{Y}}
$$

where $\mu$ is the covariance of the two variables $x_{i}$ and $y_{i} . \sigma_{x}$ and $\sigma_{y}$ are the standard deviations of $x_{i}$ and $y_{i}$ respectively and the value of $\rho$ ranges from -1 to 1 . The 
correlation coefficient $\rho$ serves as a measure of the extent to which $X$ and $Y$ are dependent. When $\rho=0$, the random variables $X$ and $Y$ are said to be uncorrelated and $\rho=-1$ or $1, X$ and $Y$ are completely independent. For the discrete case, (75) becames

$$
\rho=\frac{\sum_{i}\left(x_{i}-m_{X}\right)\left(y_{i}-m_{Y}\right)}{\left[\sum_{i}\left(x_{i}-m_{X}\right)^{2} \sum_{i}\left(y_{i}-m_{Y}\right)^{2}\right]^{\frac{1}{2}}}
$$

where $m_{X}$ and $m_{Y}$ are the mean values of $X$ and $Y$, respectively.

\section{V.2.2 ERP Analysis}

We have saved the peak values and their locations of the ERP in the vector $P_{i}$ and $T_{i}^{M F}$ in Chapter III, and we also recorded the sharpest slopes and their time locations in $S_{i}$ and $T_{i}^{W T}$ when we were processing EEG using wavelet transform in Chapter IV. So using the Equation (76), we calculate the correlation coefficient $\rho_{P S}$ for $P_{i}$ and $S_{i}$ and the correlation coefficient $\rho_{T T}$ for $T_{i}^{M F}$ and $T_{i}^{W T}$.

The results (shown in Table 4.) indicate that most of the normal subjects have relatively higher correlation coefficients for both peak and time factors than the memory-impaired subjects. Furthermore, for normal subject group, the time correlation coefficients, in average, are not as high as the ones for peaks. One of the reasons is due to the shifts caused by the smoothing function or, during the wavelet transform processing when the basic was dilated to higher scale. Another reason probably the wavelet transform picked the slopes in wrong latencies but $P_{300} / \mathrm{LPC}$. We have seen this from the mean values of the time locations of maxima and minima slopes that we obtained in Chapter IV. But however, we still can show the difference to impaired group. This means that even though we did not get the ideal results from the wavelet transform, the information that we extracted by using this two methods is still mathematically related. More experiment need to be done using this method to give the further medical explanation. 


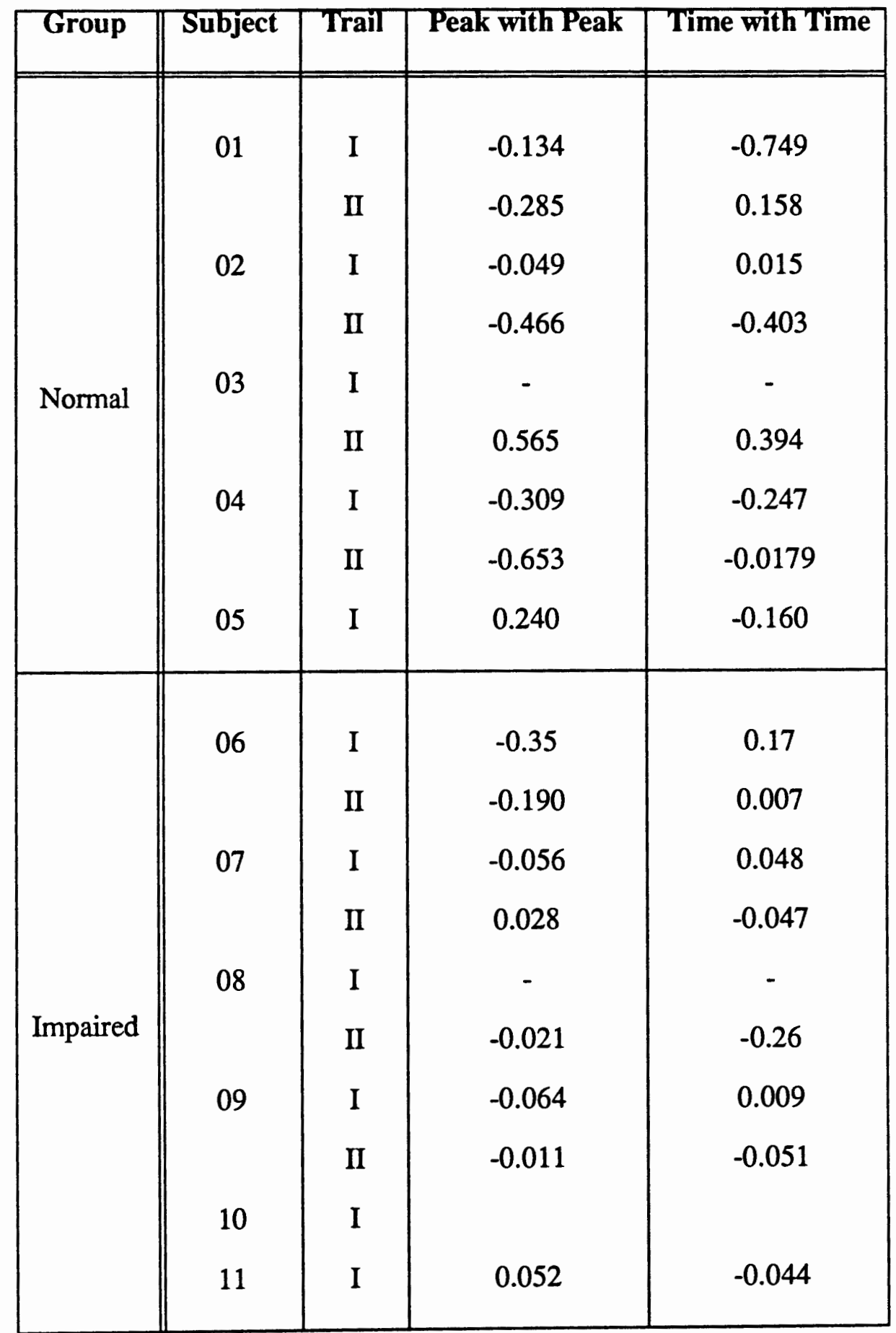

Table 4. Correlation coefficients between the outputs of the matched filter and wavelet transform. 


\section{V.3 COMPARISON}

\section{V.3.1 The Similarity between the Matched Filter and the WT}

From Chapter III, we know the the output of the signal $f(t)$ of the matched filter is

$$
g(t)=f(t) * f\left(t_{m}-t\right)
$$

and from the Equation (72) in Chapter IV, the spline wavelet transform of signal $f(t)$ can be written as

$$
W_{s} f=s \frac{d}{d t}\left(f * \theta_{s}\right)(t) .
$$

If we only consider the first step of the wavelet transform, $f * \theta_{s}$, in this thesis, the wavelet transform and matched filter are both designed to choose a window function to smooth the raw signal, attenuating the high frequencies components. It was the reason that the output of the matched filter and the smoothing output of wavelet transform at scale $2^{4}$ are so similar to each other. Figure 21(a) is EEG signal, Figure 21(b) is its output of matched filter and Figure 21(c) is the smoothed part of wavelet transform at scale $s^{4}$. So why we obtained fairly consistent results for both the normal group and the impaired group in Chapter III but could not obtain the consistent results in Chapter IV?

\section{V.3.2 The Differences between the Matched Filter and the WT}

1. Recall the Equation (11) in Chapter III

$$
h_{m}(t)=f\left(t_{m}-t\right) \text {. }
$$

So we know that the window of the matched filter was designed to attenuate strongly those frequency components in frequency intervals having little relative signal energy while attenuating very little those components where the relative 
signal energy is high.

The phase response is also very important and Equation (9)

$$
H_{m}(\omega)=k F^{*}(\omega) e^{-j \omega t_{m}}
$$

states that the phase shifts in $f(t)$ should be negated in such a way that all frequency components in $f(t)$ add in phase at exactly the time $t=t_{m}$. So the noise spectral components add with random phases so that the peak-signal-to-noise ratio is maximized. And we know that one of the severe problems of ERP analysis is its very low SNR. It was why the matched filter benefited the peak analysis.

The window chosen for the matched filter is 128 time points and fit for the the late positive complex of ERP's, so the output of the matched filter would match the whole late positive complex, not only the $P_{300}$ component. The peaks we detected using matched filter might not be the peak of the $P_{300}$ component but the ones for the whole late positive complex.

2. On the other hand, wavelet transform selects the frequency components by smoothing all the components within the windows. The only thing that we can choose for obtaining the interesting components is the window size. But the window design of wavelet transform, in other words, the window is a spline function, does not concern the energy distribution and the the phase shifts.

We designed the window of the wavelet to detect the The $P_{300}$ component but the $P_{300}$ component might not be the main component in the late positive complex.

3. There is another reasons that might effect the results of the ERP analysis using wavelet transform. We did not set the same threshold as the one we used in 
Chapter III. We used the normalized value 0.1 to be the threshold when we were using the matched filter method but we just simply used zero to be the threshold when we were using the wavelet transform method.

\section{V.4 CONCLUSION}

In summary, according to the characteristic of ERP waveforms, it is very important for brain wave analysis to improve the SNR ERP. Removing eyeblinks accurately may further improve the ERP analysis Even though the spline wavelets did not perform very well in EEG signal processing, we still observeed, from that correlation coefficients, that the slopes that we picked using WT were somehow related to the peaks of the outputs of the matched filter function. The applications of wavelet transform for ERP waveform analysis is just a beginning. There are many other types of wavelets that can be considered to be used for ERP analysis. Some of the applications for other type of signals, e.g. image signal and speech signal, both of them have very high SNR, have been well done. So we believe that in our further research of ERP analysis, other types of wavelet functions and/or the combinations of wavelet transform with other techniques might provide some potential applications. 

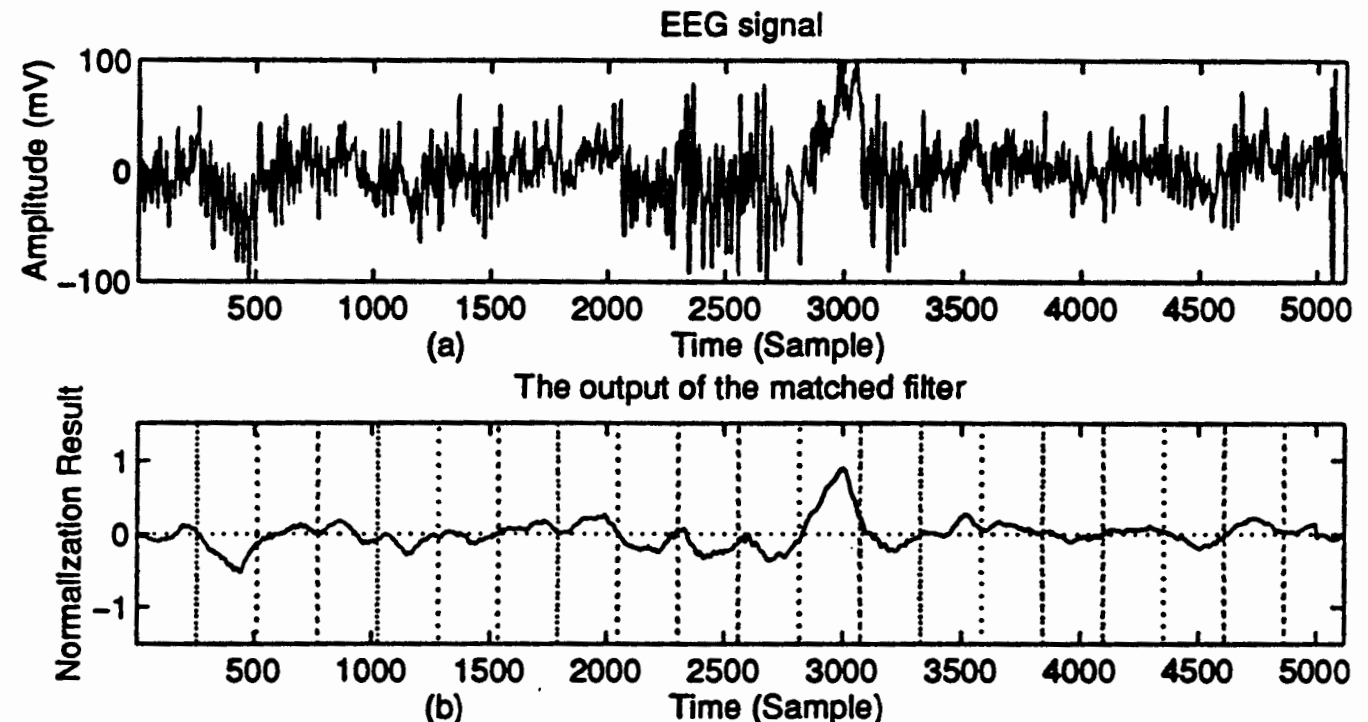

The Smoothed Part of the Wavelet Transform

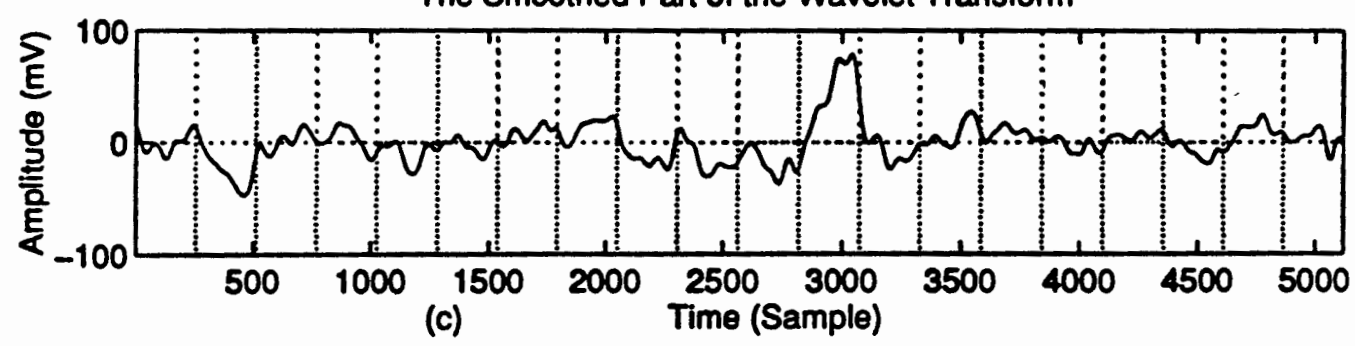

Figure 21. (a) is EEG signal, (b) is the output of the matched filter, and (c) is the smoothed part at scale $2^{4}$. 


\section{REFERENCES}

[1] Kai-Bor Yu and Clare D. Mc Gillem, "Optimum Filters for Estimating Evoked Potential Waveforms." IEEE Trans. on Biomedical Engineerring, Vol. BME-30, No. 11 pp. 730-737, November 1983

[2] Kenneth R. Erichson's research proprosal, "Event Related Potential Changes in Memory Dysfunction" pp. 6-10, December 1984.

[3] Samuel K. Law, John W. Rohrhaugh, Charles M. Adams and Michael J. Eckardt, "Improving Spatial and Temporal Resolution in Evoked EEG Responses Using Surface Laplacians." Electroencephalography and Clinical Neurophysiology, Vol. 88, pp. 309-322, 1993

[4] D. H. Lange, H. Pratt and G. f. Inbar, "Matched Filtering of Evoked Potentials: A Real Time Approach." Electroencephalography and Clinical Neurophysiology, Vol.88, pp. 187-191, March 1993.

[5] Ferrel G. Stremler, "Introduction to Communication Systems", pp. 432-435 Tk5103.S74, 1990.

[6] Olivier Rioul and Martin Vetterli, "Wavelets and Signal Processing." IEEE SP Magazine, pp. 14-18, October 1991.

[7] Ingrid Daubechies, "Ten Lectures on Wavelets", pp. 2-10, QA403.3 .D38, 1992

[8] Stephane Mallat, "Zero-Crossing of the A Wavelet Transform." IEEE Trans. on Information Theory, vol 37, No. 4, pp. 1019-1033, July 1991.

[9] Stephane Mallat and Sifen Zhang, "Characterization of Signals From Multiscale Edges." IEEE Trans. on Pattern Analysis and Machine Intelligence, Vol. 14, No. 7 pp. 710-732, July 1992.

[10] Hong Qi, "Pattern Recognition and ERP Waveform Analysis Using Wavelet transform", Thesis for the Degree of Master of Science in Electrical Engineering, Portland state university, 1994

[11] Stephane Mallat and Wen Liang Hwang, "Singularity Detection and Processing With Wavelets." IEEE Trans. on Information Theory, Vol. 38, No. 2, pp. 617-643, March 1992.

[12] Hong Qi, "Pattern Recognition and ERP Waveforn Analysis Using Wavelet Transform." A Thesis for Master of Science in Electrical Engineering, Portland 
State University. pp. 17-19, 1993

[13] Stephane Mallat, "A Theory for Multiresolution Signal Decomposition: The Wavelet Representation." IEEE Trans. on Pattern Analysis and Machine Intelligence, Vol. 11, No. 7, pp. 674-693, July 1989.

[14] Ping Wah Wong, "Wavelet Decomposition of Harmonizable Random Processes." IEEE Trans. on Information Theory, Vol. 39, No. 1, pp. 7-18, January 1993.

[15] Ingrid Daubechies, "The Wavelet Transform, Time-Frequency Localization and Signal Analysis." IEEE Trans. on Information Theory, Vol. 36, No. 5, pp. 961-1005, September 1990.

[16] Micheal Unser and Murry Eden, "On the Asymptotic Convergence of B-Spline Wavelets to Gabor Functions." IEEE Trans. on Information Theory, Vol. 38, No. 2, pp. 864-872, March 1992.

[17] Micheal Unser, Akram Aldroubi, and Murry Eden, "Fast B-Spline Transforms Representation and Interpolation." IEEE Trans. on Pattern Analysis and Machine Intelligence, Vol. 13, No. 3, pp. 277-285, March 1991.

[18] Jiann-Shu Lee, Yung-Nien Sun, Chin-Hsing Chen, and Chine-tsong Tasi, "Wavelet Based Corner Detection." Pattern Recognition, Vol. 24, No. 6, pp. 853-865, 1993.

[19] Joseph J. Liang, "A New Approach to Classification of Brainwaves." Pattern Recognition, Vol. 22, No. 6 pp. 767-774, 1989.

[20] Walton T. Roth, Judith M. Ford, and Bert s. Kopell, "Long-Latency Evoked Potentials and Reaction Time." Psychophysiology, Vol. 15, No. 1, pp. 17-23, 1978.

[21] R. A. Gopinath and C. S. Burrus, "Wavelet-based Lowpass/Bandpass Interpolation." IEEE Proc. ICASSP'92, March 1992.

[22] Jonh E. Desmedt and Vincent Chalkin, "New Method for Titrating Differences in Scalp Topographic Pattern in Brain Evoked Potential Mapping." Electroencephalography and Clinical Neurophysiology, pp. 359-366, 1989.

[23] Robert Schalkoff, "Pattern Recognition: Statistical, Structural and Neural Applications." John Wiley \& Sons Inc., 1992.

[24] H. E. Hanrahan, "A Family of Wavelets Which Are Dilatable BY Simple IIR Filters." Electrical Engineering Dept., University of the Witwatersrand Private Bag X3, Wits 2050, South Africa.

[25] Charles K. Chui and Jian-Zhong Wang, "On Compactly Supported Spline Wavelets and A Duality Principle." Transactions of the American Mathematical 
Society, Vol. 330, No. 2, pp. 903-915, April 1992.

[26] Anil K. Jain and Sushil K. Bhattacharjee, "Address Block Location On Envelopes Using Gabor Filters." Pattern Recognition, Vol. 25, No. 12, pp. 1459-1477, 1992.

[27] R. Mehrotra, K. R. Namuduri, and N. Ranganathan, "Gabor Filter-Based Edge Detection." Pattern Recognition, Vol. 25, No. 12, pp. 1479-1494, 1992.

[28] E. A. Bartnik, K. J. Blinowska, and P. J. Durka, "Signal Evoked Potential Reconstruction by Means of Wavelet Transform." Biol. Cybern. Vol. 67, pp. 175-181, 1992.

[29] Tim Edwards, "Discrete Wavelet Transform: Theory and Implementation" Stanford University, September 1991.

[30] Vinvent J. Samar, Mysore R. Raghuveer, "Wavelet Decomposition of Event Related Potentials: Toward the Definition of Biologically Natural Components" Rochester Instutute of Technology and University of Rochester Medical Center

[31] Letter, "Wavelet--New Method of Evoked Potential Analysis" Med. \& Biol. Eng \& Compute., Vol. 30, pp. 125-126, 1991 